\title{
Empirical Models of the Communications Performance of Multi-hop Cellular Networks using D2D
}

\author{
B. Coll-Perales, J. Gozalvez and M. Sepulcre \\ UWICORE, Ubiquitous Wireless Communications Research Laboratory, http://www.uwicore.umh.es \\ Miguel Hernandez University of Elche, Avda. de la Universidad, s/n, 03202 Elche, Spain \\ bcoll@umh.es, i.gozalvez@umh.es, msepulcre@umh.es
}

Corresponding author:

B. Coll-Perales

Miguel Hernandez University of Elche

Avda. de la Universidad, s/n, 03202, Elche, Spain

E-mail: bcoll@umh.es

Phone: +3496522 2031 / Fax: +34 +34966 658903

\begin{abstract}
Multi-hop Cellular Networks (MCNs) can improve the quality of service, capacity and energy-efficiency of traditional infrastructure-centric single-hop cellular systems. MCN systems can exploit Device-to-Device (D2D) communications and utilize the communications and computing capabilities of mobile devices. However, the communications challenges resulting from the use of mobile devices and D2D communications require empirical solid evidences of their performance benefits, and the design of robust communications and networking protocols. In this context, this paper presents a unique set of empirical models of the communications performance of MCN systems that utilize D2D communications. The models take into account the impact of the distance, propagation/visibility conditions, number of hops, and communication settings. The set of derived models can help design, test and optimize communications and networking protocols for MCNs that utilize D2D communications.
\end{abstract}

Keywords- Multi-hop cellular networks; device-to-device; D2D; mobile relays; empirical models; field trials; 5G; device-centric wireless networks.

\section{Introduction}

Cellular systems have significantly evolved over the past decades through the introduction of novel radio access technologies designed to increase capacity and support higher data rates. The evolution has mainly focused around the traditional infrastructure-centric approach where each Mobile Station (MS) directly communicates with the Base Station (BS). Traditional cellular communications experience difficulties in providing high and homogeneous Quality of Service (QoS) levels throughout the cell (in particular at cell edges) due to the signal attenuation produced by obstacles and distance. Future $5 \mathrm{G}$ networks will also require novel solutions to support the expected growth (by a factor of 500 to 1000) in mobile traffic in the next decade 
[1]. These forecasts have launched the race towards the design of 5G. Relevant efforts focus on the use of higher frequency bands, the dense deployment of small cells, and the design of advanced transmission technologies. These approaches build from traditional cell-centric architectures. An alternative is the emergence of device-centric wireless networks that exploit the intelligence, communications and computing resources of smart mobile devices [2]. Device-centric wireless networks have been fostered by the identified benefits of Device to Device (D2D) communications that facilitate new value added services (including proximity based services), support critical public safety applications, help offload cellular traffic from the base stations, and increase the spatial frequency reuse and the capacity of cellular networks. Future device-centric wireless networks will enable mobile devices to provide wireless connectivity to other devices, and hence act as a bridge with the cellular infrastructure. The integration of cellular and ad-hoc or D2D communications is referred to as Multi-hop Cellular Networks (MCN). MCNs will transform mobile devices into prosumers of wireless connectivity in an underlay network that if efficiently coordinated with the cellular network has the potential for significant capacity, energy-efficiency and Quality of Service (QoS) benefits [3]. 3GPP is already working on the design of D2D communications and proximity-based services [4]. In this context, it is important noting that 3GPP considers cellular (e.g. LTE-Direct) and 802.11 technologies for D2D communications. 3GPP TR 22.803 also covers multi-hop cellular communications between a BS and an end-user using D2D communications and mobile users as relays ('ProSe UE-to-Network Relay' in [4]).

Analytical and simulation studies have reported the benefits and advantages that MCN using D2D communications can provide over traditional cellular architectures in terms of capacity, coverage, infrastructure deployment cost, power saving and energy efficiency ([5], [6]). First experimental studies have recently demonstrated the gains achieved by MCNs using D2D communications with regards to end-user QoS and link quality when operating at large distances to the serving BS, in indoor environments, and under Non-Line-Of-Sight (NLOS) propagation conditions [3]. These gains result from the substitution of long-distance (and usually NLOS) single-hop cellular links by shorter distance (LOS) links with improved link budgets. Field tests have also demonstrated how MCNs using D2D communications can improve the end-user QoS in cell-overlaid or handover areas [7].

Field tests have provided very valuable data to evaluate and characterize the performance of MCNs using D2D communications. Field test measurements can also be used to derive models useful to the community. In this context, this study presents models of the communications performance achieved with MCNs that utilize D2D communications. The models take into account the impact of distance, propagation/visibility conditions, number of hops and communication settings. The proposed models can help design, test and optimize in analytical and simulation studies, novel communications and networking protocols tailored for MCNs. For example, the derived models could help design:

- Techniques to integrate MCNs in heterogeneous wireless networks. Future cellular/wireless ecosystems will be characterized by the coexistence of multiple but complementary radio access technologies [8]. Mechanisms are necessary to select the most adequate technology at each point in time. The inclusion of MCNs in heterogeneous wireless networks needs to take into account both their benefit (e.g. QoS, capacity, and energy-efficiency) and cost (e.g. communications overhead). In this 
context, the models here presented could help identify the conditions under which MCNs would be a viable communications alternative in heterogeneous wireless networks.

- Routing protocols for MCNs using D2D communications. MCNs networks utilizing D2D communications require establishing a path between source and destination using intermediate mobile devices to forward the information [9], [10]. The selection of forwarding nodes can strongly influence the end-to-end performance. The proposed models could help select relay nodes that ensure reliable routes that guarantee the target end-to-end performance. The models can also be used to compare the performance of different routing protocols.

- Opportunistic networking in MCNs using D2D communications. Opportunistic networking mechanisms can be designed to exploit the delay tolerance of mobile data services in order to reduce the energy consumption and improve the network's capacity [11], [12]. These mechanisms need to determine the conditions and time instant at which mobile devices storing information could forward it to the final destination. The proposed models can be used to identify the conditions under which opportunistic networking schemes can help improve the operation and performance of MCNs.

The rest of the paper is organized as follows. Section 2 presents the experimental testbed utilized to measure and characterize the communications performance of MCNs using D2D communications. Section 2 also describes the conditions under which the field trials have been conducted. Section 3 proposes an empirical model for the communications performance 2-hop MCNs using D2D communications. The modeling process is then extended to a larger number of hops in Sections 4 and 5. Finally, Section 6 summarizes the main contributions of this study.

\section{Experimental testbed and conditions}

The empirical communications performance models have been derived using the mHOP experimental testbed fully developed at the UWICORE laboratory of the Miguel Hernandez University of Elche in Spain. To the authors' knowledge, mHOP is the first testbed using live cellular networks for the study of MCNs using mobile relays and D2D communications. mHOP was developed to investigate the benefits of MCNs over traditional single-hop cellular systems, and the conditions under which such benefits can be obtained [3]. As a result, the platform includes MCN and conventional single-hop cellular links. Fig. 1 illustrates the mHOP architecture, and shows how the destination Mobile Node (MN) can be reached through a single-hop cellular connection, or an MCN connection (the platform focuses on downlink transmissions). The MCN connection requires access to the cellular infrastructure through a hybrid MN capable of forwarding cellular data in real-time to the destination $\mathrm{MN}$ using other MNs and D2D communications. 


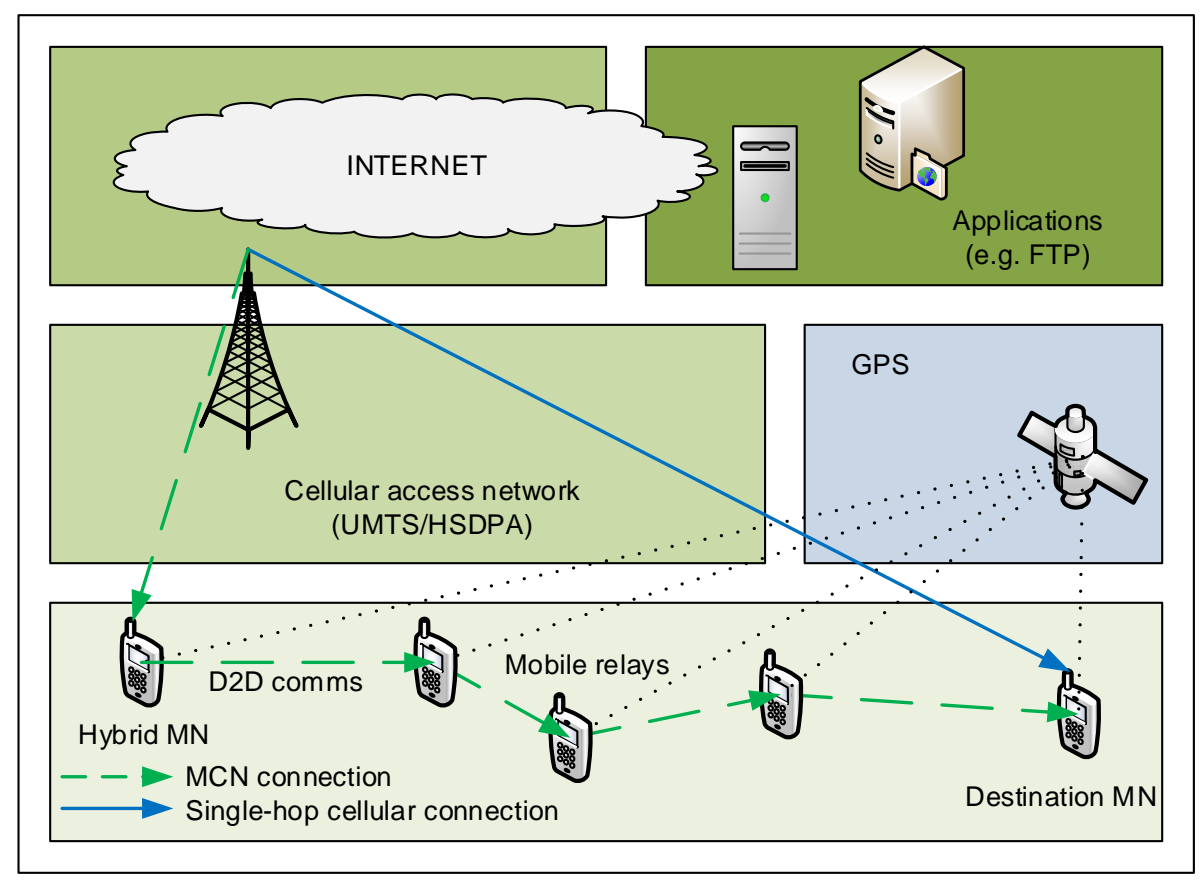

Fig 1. mHOP architecture.

\subsection{Cellular Connectivity}

The mHOP platform includes cellular connectivity into the hybrid $\mathrm{MN}$ and the destination $\mathrm{MN}$ using a Nokia 6720c handset. The terminal supports HSPA (category 9), and incorporates the Nemo Handy application (http://www.anite.com). Nemo Handy is a professional network testing tool used to extensively monitor in real-time the operation of cellular networks. It provides a large set of parameters and measurement data captured over voice and video calls, and FTP/UDP data transfers, among others. Nemo Handy also allows storing the monitored parameters for post-processing using Nemo Outdoor (and Nemo Analyze). Nemo Outdoor offers a valuable set of key performance indicators, e.g. throughput, BLER (Block Error Rate) or RSSI (Received Signal Strength Indication). Another important feature of Nemo Handy is the possibility to lock the cellular connection to a specific radio access technology and BS. This feature facilitated a more stable testing environment in order to compare single-hop cellular and MCN connections. Finally, Nemo Handy also provides spatial and time synchronization through an external GPS connected via Bluetooth. The GPS data has been used to track the MS position and geo-reference all the performance measurements.

\subsection{D2D Connectivity}

mHOP Mobile Relays (MRs) have been implemented over Linux laptops, and use IEEE 802.11 for their D2D transmissions ${ }^{1}$. In addition to their built-in wireless interface, the laptops have been equipped with a Wireless ExpressCard integrating the AR9280 Atheros chipset. The laptops use their Wireless ExpressCard for D2D transmissions, and they monitor the transmitted and received packets using both their built-in wireless interface and a virtual interface created using the Wireless ExpressCard. The laptops have also been equipped with

\footnotetext{
${ }^{1}$ 3GPP considers IEEE 802.11 technologies as well as cellular technologies (e.g. LTE-Direct) for device-todevice communications [4]. At the time this study was conducted, LTE-Direct was not yet released and available in commercial off-the-shelf mobile devices.
} 
an USB GPS receiver that, together with the Network Time Protocol (NTP), allows time-synchronizing all $\mathrm{mHOP}$ devices.

The MRs operate under Linux using the Ubuntu distribution. This solution was chosen due to the availability of open tools to configure parameters at the 802.11 physical and MAC layers. In particular, mHOP uses the Backports and iw packages to configure the wireless interfaces. Backports includes the Ath9k driver used to control the Wireless ExpressCard. Using the iw package, it is possible to configure the transmission channel and power, as well as the data rate of the Wireless ExpressCard. The iw package is also used to configure the Wireless ExpressCard in ad-hoc mode, create virtual wireless interfaces, and configure the built-in and virtual interfaces in monitor mode. The iw package is used to create the ad-hoc network that mHOP MRs will join to establish the MCN end-to-end connection. The MRs incorporate a packet sniffer software developed at UWICORE to capture 802.11 traffic. The sniffer uses the open source Linux libpcap library to extract information from the 802.11 packets' header (radiotap), e.g. their timestamp, RSSI, data rate, frequency channel, packet size, or frame check sequence. Having access to the raw captured packets allows a much faster and customized filtering and post-processing than using certain network analyzer tools such as Wireshark or Tshark. The MRs time and geo-reference all the captured packets using the USB GPS module and a simple application developed to collect the time, latitude and longitude with a refresh periodicity of $1 \mathrm{~Hz}$.

The hybrid MN is an MR that also acts as a gateway between the cellular and D2D links. To this aim, the hybrid $\mathrm{MN}$ is also implemented on a laptop with all previously described 802.11 features, and uses a Nokia 6720c terminal as modem to provide the cellular link required in MCN connections. The hybrid $M N^{\prime}$ routing table has been modified to allow for the real-time forwarding of information from the cellular to the D2D links. The hybrid MN includes two GPS receivers and the software monitoring tools previously described.

\subsection{Testing Environment}

The field tests have been conducted in the city of Elche (Spain) using Orange's live cellular network and the mHOP testbed. The field tests investigate the impact of propagation conditions, distance and number of hops, and communication settings on the performance of MCNs using D2D communications. Tests are conducted for different distances between the Hybrid MN (HN) and the BS. During the tests, the MRs and the Destination MN (DN) vary their locations depending on the number of hops and the conditions under study. The field tests were conducted in a long and straight four-lane avenue close to the University. The avenue ensured LOS conditions between the HN and the MRs when they were located at the same side of the avenue. The NLOS conditions were achieved locating the HN and the MRs in opposite sidewalks of the avenue, with vehicles continuously crossing over and dense vegetation in the avenue's median.

The tests start when all nodes execute a script that establishes the cellular connection from the $\mathrm{HN}$ to its serving BS, and the D2D links between the MRs. During the tests, the iperf network testing tool is used to transfer UDP packets ${ }^{2}$ from a server managed and located at

\footnotetext{
${ }^{2}$ UDP traffic was used since it allows indentifying the radio link that represents the bottleneck of a multi-hop transmission. This feature was very valuable to analyze separately the performance of various links of a MCN connection.
} 
the UWICORE lab to the mobile DN. Iperf allows specifying the size of the UDP packets that were set to the largest allowed Ethernet size at the network layer, i.e. 1548 bytes including headers. The cellular connection uses HSPA as LTE was not available in Elche at the time these field tests were conducted ${ }^{3}$. D2D links are established using IEEE 802.11g. This technology was selected over IEEE802.11a to benefit from the better propagation conditions experienced at $2.4 \mathrm{GHz}$ compared to $5 \mathrm{GHz}$ [13]. IEEE $802.11 \mathrm{~g}$ provides a theoretical maximum bit rate of $54 \mathrm{Mbps}$ and defines a maximum transmission power of $27 \mathrm{dBm}$. However, the transmission power was limited to $19 \mathrm{dBm}$ in compliance with the Spanish regulations (limit is established at $20 \mathrm{dBm}$ ). Unless otherwise specified, the data rate and transmission power in D2D links were dynamically modified based on the link quality conditions and the adaptive mechanisms implemented in the 802.11 cards' driver. Additional field tests were conducted fixing the data rate and the transmission power.

\subsection{Performance Metric}

The communications performance of the single-hop and MCN connections is measured in terms of the throughput experienced by the mobile DN. In the case of single-hop cellular transmissions, it would have been reasonable to expect that as the DN moves away from the BS and the link quality degrades (measured in HSPA through the Channel Quality Indicator CQI - parameter), the throughput decreases. However, initial tests showed that the operator's radio resource management policy compensated such link degradation with more radio resources. In particular, the network increased the percentage of time that radio resources were used (referred to as usage) by users experiencing poor link quality conditions. This resulted in that, for example, a MS located close to the BS (reporting high channel quality) could experience the same user throughput as a MS located at the cell edge (reporting low channel quality) if the usage was higher for the user located at the cell edge. It is important noting that different operators/networks might implement different radio resource management policies, and that such policies were not under control of the authors. To reduce the impact of the radio resource management policies on the conducted study, and capture the dependence of the cellular performance with the channel quality, the throughput performance (whether single-hop cellular or MCN) is here computed as the ratio between the measured throughput and the usage parameter. In the previous example, the selected metric results in that the MS located close to the BS would experience a higher throughput than the MS located at the cell edge.

\section{2-Hop MCN Communications}

First models of the communications performance for 2-hop MCN communications were presented in [14] that are here extended. This study considers the scenario illustrated in Fig. 2. During the tests, the HN remains in a static position, while the DN moves away from the location of the HN until the D2D link with the HN is lost. Various locations of the $\mathrm{HN}$ at different distances from the BS have been analyzed. Field tests have been conducted for LOS and NLOS conditions between the HN and DN nodes.

\footnotetext{
${ }^{3}$ The study though defines the conditions to extend the derived models to other cellular technologies.
} 


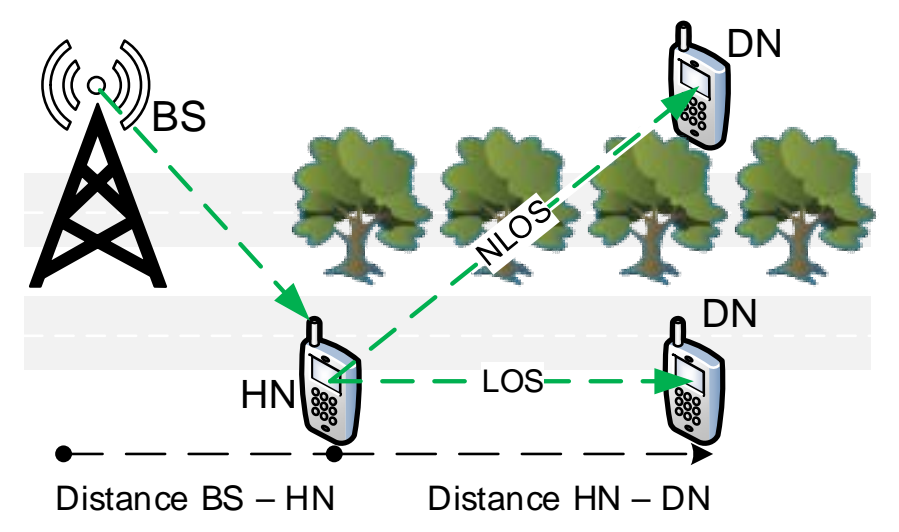

Fig 2. Testing environment for 2-hop MCN communications.

\subsection{Field Tests}

Fig 3.a shows two examples of the throughput experienced at the DN for the 2-hop MCN connection when the HN-DN D2D link is characterized by LOS conditions. The HN was located 200 meters (left figure) and 500 meters (right figure) away from the BS. The throughput is depicted as a function of the distance between the HN and the mobile DN (the DN begins the tests close to the HN). The MCN throughput at the DN is first upper-bounded by the cellular performance of the BS-HN link $\left(\mathrm{Thr}_{H N}\right)$ for short HN-DN distances. The $802.11 \mathrm{~g}$ throughput performance of the D2D link between $\mathrm{HN}$ and DN is high when the distance between the two nodes is small. In this case, the MCN throughput at the DN is upper bounded by the lower throughput experienced in the BS-HN cellular link. The performance at the mobile DN starts decreasing when the D2D link quality starts degrading as the distance between HN and DN increases (this distance will be referred to as critical distance). This happens at an approximate distance between $\mathrm{HN}$ and DN of 140-160 meters. From this distance onwards, the MCN throughput at the DN of the 2-hop MCN connection is upper-bounded by the D2D performance. Similar trends are observed under NLOS conditions (Fig. 3.b). The main difference lies in the HN-DN distance from which the throughput at the mobile DN decreases (approximately 45 meters). This difference is explained by the more challenging NLOS propagation conditions between $\mathrm{HN}$ and $\mathrm{DN}$, and the consequent lower received signal levels.

A large set of field trials have been conducted for various HN locations. In particular, trials have been conducted with the HN located 200, 500 and 800 meters away from the BS. Varying the location of the HN resulted in different average HSPA throughput levels at the HN and MCN throughput performance at the mobile DN. However, similar trends to that depicted in Fig. 3 were always observed. 

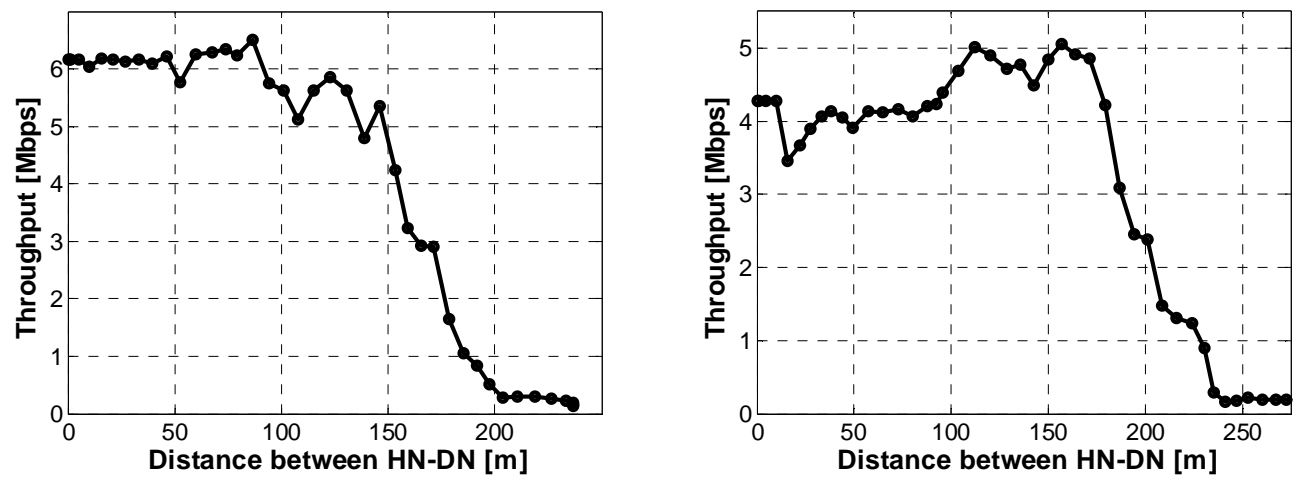

a) HN-DN link under LOS conditions.
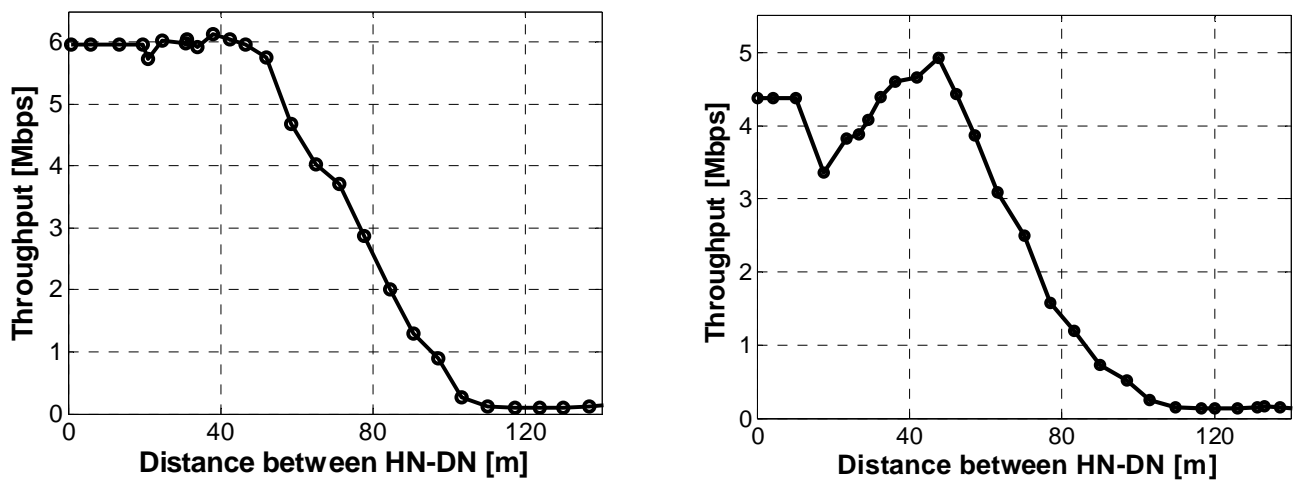

b) HN-DN link under NLOS conditions.

Fig. 3. 2-hop MCN throughput at the mobile DN with HN located 200 (left) and 500 (right) meters away from the BS.

\subsection{Modeling Functions}

The first step to derive performance models for MCNs using D2D communications is to identify the functions that best fit the throughput measured at the mobile DN of the 2-hop MCN connection. This process is based on an average processing of filed measurements like those reporting in Fig. 3. The performance depicted in Fig. 3 has a symmetric $S$ shape. There are several mathematical functions that can model such shape, and that can be grouped into three broad categories: sigmoid, piecewise-defined and exponential functions. A function from each of these categories has been selected based on the possibility to adequately fit their expression to the field measurements, and the simplicity of their expression for facilitating future analytical and simulation uses. More complex functions could also provide good fits, but the selected ones provide a reasonable trade-off between accuracy and analytical-simulation tractability. In particular, the following functions have been selected:

- The Sig function (1) has asymptotes $y=A$ and $y=0$, and is symmetric at $d=p_{2}(d$ represents the distance in meters). The parameters $A$ and $p_{1}$ determine the maximum slope $\left(A \cdot p_{1} / 4\right)$ of the Sig function at $d=p_{2}$.

$$
\operatorname{Sig}\left(p_{1}, p_{2}, d\right)=A \cdot\left[1-\frac{1}{1+e^{-p_{1} \cdot\left(d-p_{2}\right)}}\right], \forall d \in\{0, \ldots, M\}
$$

To calculate the distance $d$ at which the Sig function decreases below $y=A$ (critical distance), it is first necessary to define the maximum curvature point or upper knee 
$\left(K_{u}\right)$ of the Sig function [15]. This parameter can be calculated using the curvature (Curv) function:

$$
\operatorname{Curv}(d)=\frac{\operatorname{Sig~}^{\prime \prime}(d)}{\left(1+\left(\operatorname{Sig}^{\prime}(d)\right)^{2}\right)^{3 / 2}}=\frac{\frac{A \cdot p_{1}{ }^{2} \cdot t \cdot(1-t)}{(1+t)^{3}}}{\left(1+\left(\frac{-A \cdot p_{1} \cdot t}{(1+t)^{2}}\right)^{2}\right)^{3 / 2}}
$$

where $t=\exp \left(-p_{1} \cdot\left(d-p_{2}\right)\right)$. Computing the critical distance also requires defining the utility $(U)$ function:

$$
U(d)= \begin{cases}\operatorname{Sig}(d)-P(d) & d \leq K_{u} \\ \operatorname{Sig}(d) & d>K_{u}\end{cases}
$$

In (3), $P(d)$ is a monotonically increasing function $\left(P(d)=\alpha \cdot\left(d-K_{\mathrm{u}}\right)\right)$ that represents the penalty assigned to $d \leq K_{u}$. The critical distance is then obtained by setting $U^{\prime}(d)=0$ and solving the equation $\operatorname{Sig}^{\prime}(d)=P^{\prime}(d)=\alpha$ where $|\alpha|<\left|\operatorname{Sig}^{\prime}\left(K_{u}\right)\right|$.

- The Part function (4) is defined by the asymptotes $y=A$ and $y=0$ when $d$ is smaller than $p_{1}$ and higher than $p_{2}$ respectively. Otherwise, Part function is characterized by a slope equal to $\left(-A /\left(p_{2}-p_{1}\right)\right)$. The Part function is symmetric in $d=\left(p_{2}-p_{1}\right) / 2$ and its critical distance is located at $p_{1}$.

$$
\operatorname{Part}\left(p_{1}, p_{2}, d\right)= \begin{cases}A & d<p_{1} \\ k \cdot\left(\frac{1}{d}-\frac{1}{p_{2}}\right) & p_{1} \leq d<p_{2}, \quad \forall d \in\{0, \ldots, M\} \\ 0 & p_{2} \leq d\end{cases}
$$

$$
\text { where } k=A /\left(1 / p_{1}-1 / p_{2}\right)
$$

- The Exp function (5) is defined as:

$$
\operatorname{Exp}\left(p_{1}, p_{2}, d\right)=A \cdot e^{-\left(p_{1} \cdot d\right)^{p_{2}}}, \forall d \in\{0, \ldots, M\}
$$

Following a similar analysis to that conducted for the Sig function, it is possible to determine the critical distance for the Exp function.

The three selected functions are defined by the parameters $d, A, p_{1}$ and $p_{2}$. When applied to modeling the throughput of the MCN connection, $d$ is defined as the distance between the HN and the mobile DN. A represents the average cellular throughput measured at the $\mathrm{HN}\left(\overline{T h r_{\mathrm{HN}}}\right)$ for distances smaller than the critical distance. Finally, $p_{1}$ and $p_{2}$ are the variables or fitting parameters that modify the characteristics of the S-shaped functions:

1. Length of the upper asymptote (i.e. the distance at which the MCN throughput starts decreasing or critical distance).

2. Slope of the curve (i.e. how fast the $M C N$ throughput decreases with the distance from the critical distance point).

3. The start of the lower asymptote (i.e. the distance at which the MCN throughput is negligible because the D2D connectivity is lost). 
Curve fitting is one of the common techniques used to derive the fitting parameters of a modeling function from empirical data. This method seeks minimizing the residue/difference between the measured data and the proposed modeling function. Different estimators can be used to calculate the residue. The least-square estimator produces the maximum-likelihood estimate of the parameters when the residues are distributed along the modeling function [16], which is actually the case for the MCN field measurements here reported. In this context, two different curve fitting methods using least-square estimator are here used to derive the $\left(p_{1}, p_{2}\right)$ parameters that best fit the selected functions (Sig, Part and Exp) to the MCN throughput measured at the DN.

\subsubsection{Least-Squares Parameter Estimation}

The Least-Squares Parameter Estimation (LSPE) technique seeks minimizing the residue $e$ :

$$
e_{j}=\sqrt{\frac{\sum_{d=d_{1}}^{d_{M}}\left(f_{j}\left(p_{1}, p_{2}, d\right)-g(d)\right)^{2}}{M}}, j \in\{\text { Sig, Part, Exp }\}
$$

where $f_{j}\left(p_{1}, p_{2}, d\right)$ represents the modeling functions, and $g(d)$ the measured data (in this case, the MCN throughput experienced at the mobile $\left.\mathrm{DN}^{4}\right)$. LSPE derives the fitting parameters $\left(p_{1}^{i}\right.$, $\left.p_{2}^{i}\right) j$ that minimizes the residue $e_{j}^{i}$ for each of the selected functions $j(j \in\{$ Sig, Part, Exp $\})$ and the measurement test $i$.

Fig. 4 depicts an example showing that LSPE is capable to achieve a good match between the MCN throughput at the mobile DN and the selected functions under LOS and NLOS conditions ${ }^{5}$. The LSPE scheme has been applied to all field measurements, which results in a set of fitting parameters $\left(p_{1}^{i}, p_{2}^{i}\right)_{j}$ and residues $e_{j}^{i}(\forall i \in\{1, \ldots, T\}$, with $T$ representing the number of conducted field tests (in total, more than 100 field measurements were conducted). For each of the selected functions, the results are grouped based on the visibility conditions between the $\mathrm{HN}$ and the DN, and the different locations of the HN. The average of the resulting fitting parameters is shown in Table 1 . Clear differences are appreciated between the $\left(p_{1}, p_{2}\right)$ parameters obtained under LOS and NLOS conditions. However, only small differences are obtained for the different locations of the HN despite the fact that the HN throughput varies with the distance to the BS (Fig. 5). For example, the difference is smaller than 10 meters for the Part function (Table 1). As a result, the fitting $\left(p_{1}, p_{2}\right)$ parameters for the different locations of the static HN have been grouped. Table 2 shows the resulting average fitting parameters $\left(p_{1}\right.$, $p_{2}$ ) under LOS and NLOS conditions for each of the selected functions. Tables 1 and 2 also depict the average residues $e_{j}^{i}\langle\langle e\rangle$ ) between the selected functions (when using the derived fitting parameters shown in Tables 1 and 2) and the measured data under LOS and NLOS conditions. The reported results show that the Sig function provides the lower differences with the measured data under LOS and NLOS conditions.

\footnotetext{
${ }^{4}$ The measurements are averaged over time periods of $1 \mathrm{~s}$ since the GPS has an updating rate of $1 \mathrm{~Hz}$, and are processed using a simple moving average scheme with 5 samples [17].

${ }^{5}$ The measurements analyzed in Fig. 4 correspond to those reported in Fig. 3 with the HN located 200m away from the BS.
} 

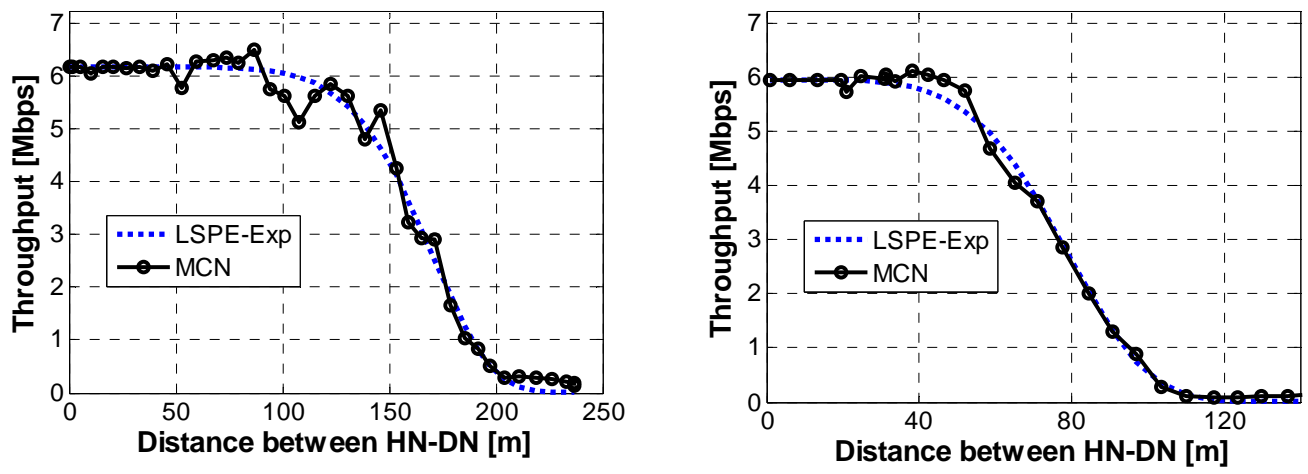

a) LSPE fitting with Exp function.
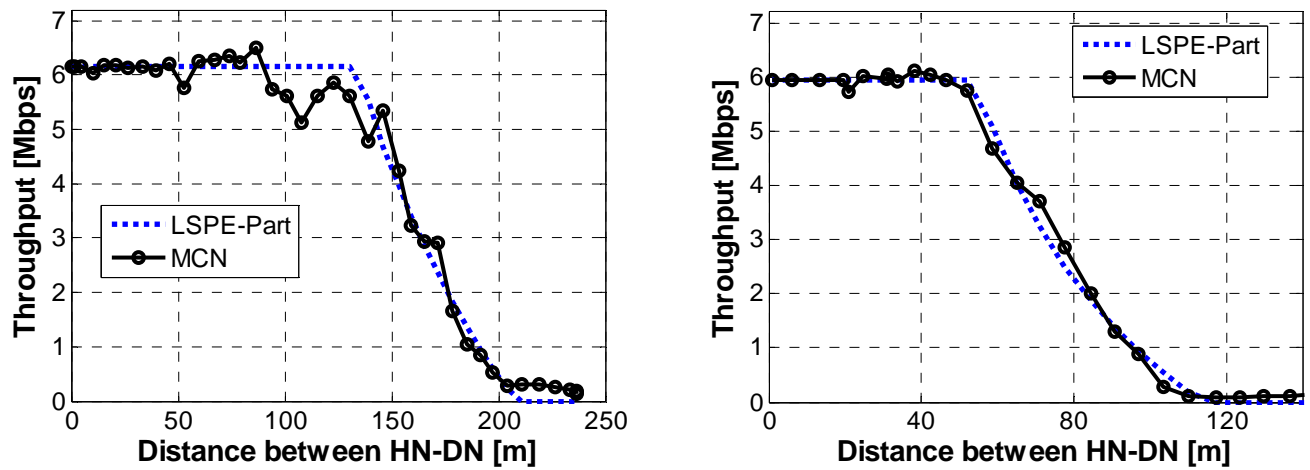

b) LSPE fitting with Part function.
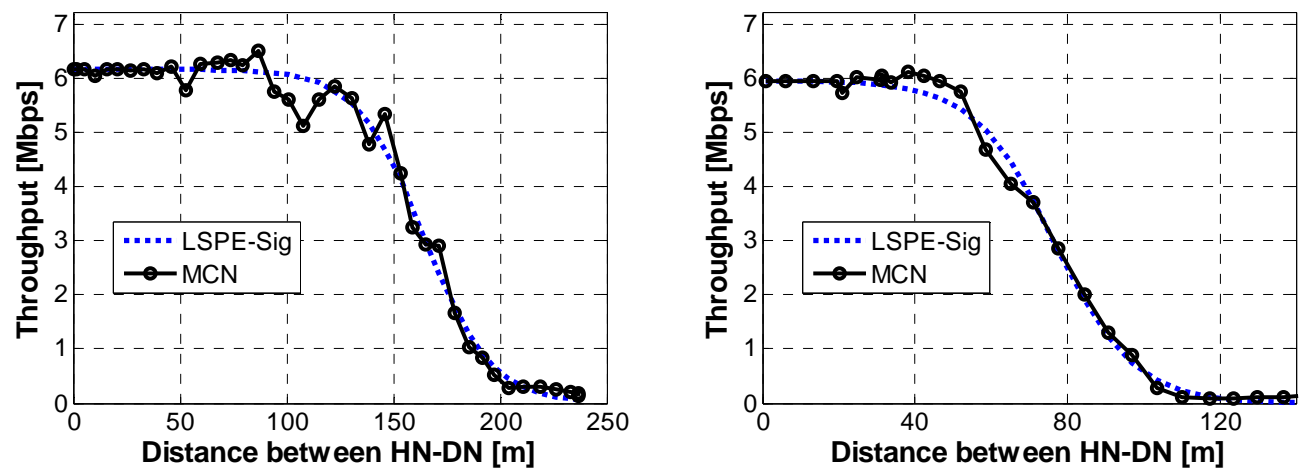

C) LSPE fitting with Sig function.

Fig. 4. LSPE fitting of the 2-hop MCN throughput measured at the mobile DN as it moves away from the location of the HN under LOS (left) and NLOS (right) conditions.

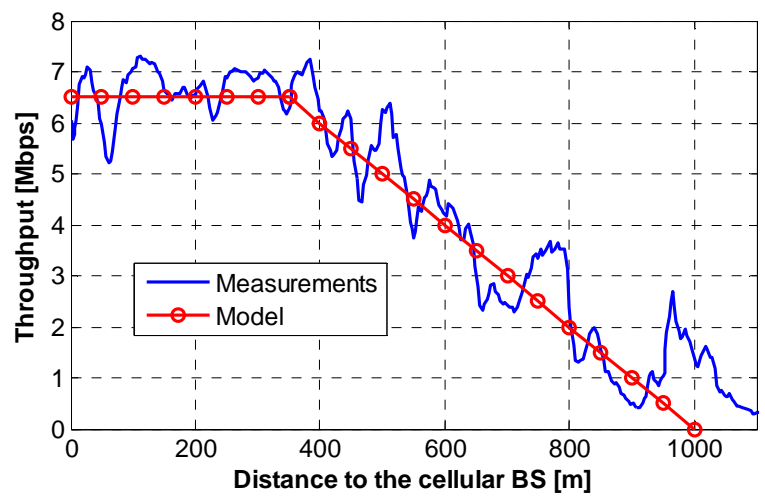

Fig. 5. HSPA throughput. 
Table 1. 2-hop MCN LSPE $\left(p_{1}, p_{2}\right)$ as a function of LOS/NLOS conditions and distance of HN to the serving BS (in meters).

\begin{tabular}{|c|c|c|c|c|c|c|c|}
\hline & \multicolumn{6}{|c|}{ Function } \\
\hline & & \multicolumn{2}{|c|}{$\operatorname{Exp}$} & \multicolumn{2}{|c|}{ Part } & \multicolumn{2}{|c|}{ Sig } \\
\hline & & $\left(p_{1}, p_{2}\right)$ & $\langle e\rangle[\mathrm{Mbps}]$ & $\left(p_{1}, p_{2}\right)$ & $\langle e\rangle[\mathrm{Mbps}]$ & $\left(p_{1}, p_{2}\right)$ & $\langle e\rangle$ [Mbps] \\
\hline \multirow{3}{*}{ อิ } & z 200 & $(0.005,11.46)$ & 0.146 & $(156.5,234.6)$ & 0.146 & $(0.107,177.6)$ & 0.140 \\
\hline & 500 & $(0.005,13.8)$ & 0.119 & $(162.1,232.5)$ & 0.114 & $(0.12,194.9)$ & 0.107 \\
\hline & 苟 800 & $(0.005,13.98)$ & 0.175 & $(151.7,229.2)$ & 0.143 & $(0.12,179.8)$ & 0.142 \\
\hline \multirow{3}{*}{$\stackrel{\text { ֻ }}{z}$} & 고 200 & $(0.012,6.44)$ & 0.061 & $(58.9,102.6)$ & 0.057 & $(0.13,75.96)$ & 0.055 \\
\hline & 500 & $(0.011,6.57)$ & 0.119 & $(62.4,108.2)$ & 0.114 & $(0.10,85.25)$ & 0.112 \\
\hline & 䓵 800 & $(0.012,6.9)$ & 0.078 & $(59.55,107)$ & 0.074 & $(0.12,78)$ & 0.074 \\
\hline
\end{tabular}

Table 2. 2-hop MCN LSPE $\left(p_{1}, p_{2}\right)$ as a function of LOS/NLOS conditions.

\begin{tabular}{c|cc|cc}
\hline \hline & \multicolumn{2}{|c|}{ LOS } & \multicolumn{2}{c}{ NLOS } \\
\hline Function & $\left(p_{1}, p_{2}\right)$ & $\langle e\rangle[\mathrm{Mbps}]$ & $\left(p_{1}, p_{2}\right)$ & $\langle e\rangle[\mathrm{Mbps}]$ \\
\hline Exp & $(0.005,12.05)$ & 0.129 & $(0.012,6.53)$ & 0.088 \\
Part & $(157.9,230.8)$ & 0.128 & $(60.6,113.8)$ & 0.089 \\
Sig & $(0.12,186.99)$ & 0.126 & $(0.12,79.67)$ & 0.087 \\
\hline \hline
\end{tabular}

\subsubsection{Linear Programming}

The fitting $\left(p_{1}, p_{2}\right)$ parameters can also be derived using Linear Programming (LP) techniques. LSPE calculated the fitting parameters by averaging the $\left(p_{1}^{i}, p_{2}^{i}\right)_{j}$ values. On the other hand, LP techniques derive the fitting parameters using all the measurements obtained under similar conditions (e.g. LOS or NLOS conditions). To this aim, LP techniques seek optimizing a linear objective function subject to a set of constraints. This work analyzed three common objective functions that are intended to provide the fitting parameters $\left(p_{1}, p_{2}\right)_{j}$ that minimize the residue $e_{j}^{6}$ between the selected functions $j \in\{S i g$, Part, Exp $\}$ and the measured data (i.e. the MCN throughput experienced at the mobile DN):

- Minimize the maximum (min-max) of the residues. The first LP scheme derives the fitting parameters $\left(p_{1}, p_{2}\right)_{\text {min-max }}$ that minimize the maximum residue $(e)$ between the selected function $(j \in\{$ Sig, Part, Exp $\}$ ) and the entire set of measurements obtained under similar conditions $(i \in\{1, \ldots, N\})$. The objective function can be expressed as:

$$
\min \max _{i \in\{1, \ldots, N\}} e_{j}^{i}
$$

\footnotetext{
${ }^{6} e_{j}$ has also been computed following the mean of the least-squares estimator reported in (6).
} 
Eq. (7) must be expressed as a linear equation to solve it using linear programming techniques. To this aim, a new real variable $z$, equal to $z=\max e_{j}^{i}$, is defined. The objective function and its constraints can now be expressed as:

$$
\min z ; \text { s.t }: e_{j}^{i} \leq z \forall i \in\{1, \ldots, N\} ; p_{1}, p_{2}, z \in \Re
$$

- Minimize the sum (min-sum) of the residues. The second LP scheme derives the fitting parameters $\left(p_{1}, p_{2}\right)_{\text {min-sum }}$ that minimize the sum of the residues $(e)$ between the selected function $(j \in\{S i g$, Part, Exp $\})$ and the entire set of measurements obtained under similar conditions ( $i \in\{1, \ldots, N\}$ ). The min-sum objective function shown in (9) is already linear, and no additional changes are required.

$$
\min \left(\sum_{i=1}^{N} e_{j}^{i}\right) ; \text { s.t }: p_{1}, p_{2} \in \Re
$$

- Minimize the multiplication (min-mul) of the residues. The third LP scheme computes the fitting parameters $\left(p_{1}, p_{2}\right)_{\min \text {-mul }}$ that minimize the multiplication of the residues $(e)$ between the selected function $(j \in\{S i g$, Part, Exp $\}$ ) and the entire set of measurements obtained under similar conditions ( $i \in\{1, \ldots, N\}$ ). In this case, the objective function is expressed as:

$$
\min \left(\prod_{i=1}^{N} e_{j}^{i}\right)
$$

Since (10) is not a linear equation, the following equivalent objective function is selected:

$$
\min \left(\sum_{i=1}^{N} \ln \left(1+e_{j}^{i}\right)\right) ; \text { s.t }: p_{1}, p_{2} \in \mathfrak{R}
$$

Table 3 shows the $\left(p_{1}, p_{2}\right)$ parameters obtained using the min-max, min-sum and min-mul LP techniques. The results only differentiate between LOS and NLOS conditions between the HN and the mobile DN following the observations reported in the LSPE analysis. Table 3 also shows

\begin{tabular}{|c|c|c|c|c|c|}
\hline \multirow{2}{*}{\multicolumn{2}{|c|}{ Function }} & \multicolumn{2}{|c|}{ LOS } & \multicolumn{2}{|c|}{ NLOS } \\
\hline & & $\left(p_{1}, p_{2}\right)$ & $\langle e\rangle[\mathrm{Mbps}]$ & $\left(p_{1}, p_{2}\right)$ & $\langle e\rangle[\mathrm{Mbps}]$ \\
\hline \multirow{3}{*}{$\begin{array}{l}\text { वे } \\
\text { है } \\
\text { है }\end{array}$} & Exp & $(0.0047,4.2)$ & 0.174 & $(0.011,4.6)$ & 0.105 \\
\hline & Part & $(153.5,251)$ & 0.174 & $(59,144.5)$ & 0.113 \\
\hline & Sig & $(0.03,194)$ & 0.174 & $(0.06,85)$ & 0.108 \\
\hline \multirow{3}{*}{$\begin{array}{l}\text { క్ } \\
\text { है } \\
\text { है }\end{array}$} & Exp & $(0.0051,5.6)$ & 0.137 & $(0.012,5.2)$ & 0.093 \\
\hline & Part & $(141.5,244.5)$ & 0.138 & $(56,113)$ & 0.094 \\
\hline & Sig & $(0.05,180.5)$ & 0.136 & $(0.09,77.5)$ & 0.091 \\
\hline \multirow{3}{*}{ 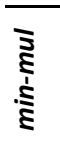 } & Exp & $(0.0053,6)$ & 0.126 & $(0.012,5.2)$ & 0.083 \\
\hline & Part & $(141,240.5)$ & 0.128 & $(56,111.5)$ & 0.084 \\
\hline & Sig & $(0.05,177)$ & 0.125 & $(0.11,77)$ & 0.081 \\
\hline
\end{tabular}
the average residues $e_{j}^{i}(\langle e\rangle)$ obtained with each of the LP schemes. The depicted results show that the Sig function minimizes $\langle e\rangle$ in most cases.

Table 3. 2-hop MCN LP $\left(p_{1}, p_{2}\right)$ as a function of LOS/NLOS conditions. 


\subsection{2-hop MCN Performance Models and Validation}

Two different methods (LSPE and LP) have been used to derive the $\left(p_{1}, p_{2}\right)$ parameters that best fit the selected functions (Exp, Part and Sig) and the MCN throughput measured at the DN. LSPE derives fitting parameters for each measurement set, and averages them all to obtain the final $\left(p_{1}, p_{2}\right)$ values. The LSPE averaging process reduces the impact of the measurement data variations that are usually observed in field tests. The impact of these variations is higher in the LP case since LP techniques take into account the entire set of measurement at once. The min-max LP technique derives the fitting parameters that minimize the maximum distance (residue) to the entire set of measurements. However, only the lowest and the highest fitting parameters have an impact on the min-max LP optimization problem, which may explain the largest average residues reported in Table 3 for the min-max scheme. The min-sum and minmul LP schemes are also influenced by measurement variations, but they only cause deviations from the general trend. These variations have a slightly larger effect in the case of the min-sum scheme since min-mul mitigates large residues $\left(e_{j}^{i}\right)$ using a logarithmic function in its objective function.

The results reported in Tables 2 and 3 show that the Sig function minimizes the average residue $\langle e\rangle$ for the LSPE and LP techniques under LOS and NLOS conditions ${ }^{7}$. The min-mul LP scheme results in the lower residue. Additional tests were carried out due to the small differences observed between LSPE and LP schemes (except for min-max). The additional tests were carried out in a different location with a different serving BS. Fig. 6 shows examples of the 2-hop MCN throughput measurements obtained at DN in these additional tests (the HN was located 200 meters away from the BS in these examples); additional tests with the HN located at different distances to the BS were also conducted. Fig. 6 also compares the measured throughput with the Sig models obtained using the fitting parameters derived through the LSPE and min-mul LP schemes. The figure shows that the Sig functions provide a good fit with the measured performance despite changing locations and serving BS. Table 4 compares the average residue $e_{i}(\langle e\rangle)$ obtained with the LSPE and min-mul LP schemes for all the additional tests conducted with a different serving BS. The reported $\langle e\rangle$ values for LSPE are in line with those listed in Table 2. However, Table 4 shows that min-mul LP derived fitting parameters perform worse when using a different BS. This can be appreciated when comparing the reported $\langle e\rangle$ values in Table 3 and Table 4 . As a result, this study proposes to model the 2-hop MCN throughput performance experienced at a mobile DN using the Sig function in (12) with the LPSE fitting parameters reported in Table 2 for LOS and NLOS conditions:

$$
\operatorname{MCN} \operatorname{Thr}\left(p_{1}, p_{2}, d\right)_{2 \text {-hop DN }}=\overline{\operatorname{Thr}} \cdot\left[1-\frac{1}{1+e^{-p_{1} \cdot\left(d-p_{2}\right)}}\right]
$$

where $d$ represents the HN-DN separation distance. For these fitting parameters, the critical distance is 156 meters and 52 meters under LOS and NLOS conditions respectively. The $\overline{T h r_{\mathrm{HN}}}$ parameter represents the average HSPA throughput measured at the HN. Fig. 5 reports HSPA throughput measurements at a node located at different distances to the BS. The throughput

\footnotetext{
${ }^{7}$ The only exception is the min-max LP scheme, but this scheme is discarded since it results in the higher residue values.
} 
depends on the actual position of the HN. Using this figure, it is possible to model the $\overline{T h r_{\mathrm{HN}}}$ throughput parameter as follows:

$$
\overline{T h r_{\mathrm{HN}}}(d)= \begin{cases}6.5 & d<350 m \\ -0.01 \cdot d+10 & d \geq 350 m\end{cases}
$$

with $d$ representing the distance between the $\mathrm{HN}$ and the serving BS. Those interested in using the 2-hop MCN throughput performance model proposed in (12) could use the $\overline{T h r_{\mathrm{HN}}}$ measurements depicted in Fig. 5 and modeled in (13), or use their own cellular measurements. It is important emphasizing that the derived model can be used for different locations of the $\mathrm{HN}$ since as previously discussed the $\left(p_{1}, p_{2}\right)$ parameters do not depend on $\overline{T h r_{\mathrm{HN}}}$.

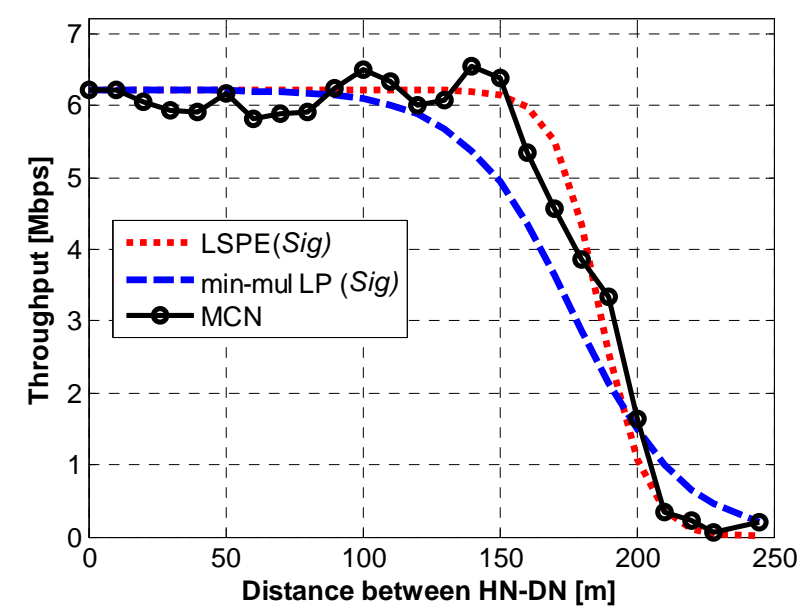

a) LOS conditions

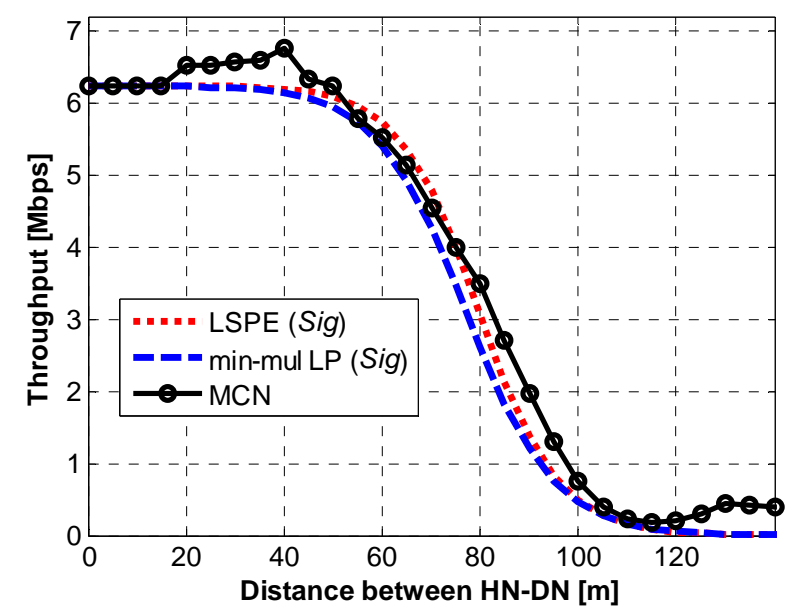

b) NLOS conditions

Fig. 6. 2-hop MCN throughput measured at the DN as it moves away from the location of the HN under LOS (a) and NLOS (b) conditions. The measurements were obtained using a different serving BS as that used for Fig. 3. The HN was located 200 meters away from the BS. The figure also shows the Sig functions derived using LSPE and min-mul LP schemes. 
Table 4. LSPE and LP comparison

\begin{tabular}{c|cc}
\hline \hline \multirow{2}{*}{ Function } & \multicolumn{2}{|c}{$\langle e\rangle$ [Mbps] } \\
\cline { 2 - 3 } & LOS & NLOS \\
\hline LSPE (Sig) & 0.132 & 0.102 \\
min-mul LP (Sig) & 0.158 & 0.110 \\
\hline \hline
\end{tabular}

\subsection{IEEE 802.11g Fixed Transmission Mode}

The previous models were obtained using the default configuration of the $802.11 \mathrm{~g}$ cards used in the mHOP testbed. This configuration adapts the transmission parameters (data rate and transmission power) based on the experienced link quality conditions. The operation of these adaptive mechanisms is driver-dependent (the mHOP testbed's 802.11g cards use the Ath9k driver), and detailed information about their implementation is generally not provided. In this context, it is of interest checking whether the 2-hop MCN throughput performance models follow similar trends when operating with fixed transmission power levels and data rates. Additional field tests have therefore been conducted under similar conditions as those reported in previous sections and illustrated in Fig. 2. The HN remains static (again, different locations of the HN were also tested with similar results), while the DN moves away from the location of the HN under LOS and NLOS conditions. The IEEE 802.11g transmission power was fixed to $19 \mathrm{dBm}(19 \mathrm{dBm}$ was previously the power limit, but the actual transmission power was dynamically modified by the 802.11 cards). The tests were conducted using the 54 and $48 \mathrm{Mbps}$ 802.11g data rates. The obtained measurements showed indeed similar trends as those observed when the $802.11 \mathrm{~g}$ adaptive scheme was active. As a result, the same LPSE curve fitting process using the Sig function has been applied to these measurements. Fig. 7 represents the derived model with the obtained fitting parameters and the average residue $(p 1, p 2,\langle e\rangle)$ for each plotted configuration. The MCN throughput curves have been obtained using the model presented in (12). Following the procedure described in Section 3.2, it is possible to calculate the critical distance, or distance at which the MCN throughput operates below the $\overline{T h r_{\mathrm{HN}}}$. The analysis reveals that when the mobile nodes communicate using the $54 \mathrm{Mbps}$ data rate, the critical distance is equal to 125 (LOS) and 50 (NLOS) meters. The $48 \mathrm{Mbps}$ data rate increments the critical distance to 143 (LOS) and 65 (NLOS) meters. In addition, the decreasing slope of the MCN throughput at the DN (related with the $p_{1}$ parameter) is greater when the mobile nodes use the $54 \mathrm{Mbps}$ data rate than when using the $48 \mathrm{Mbps}$ one. This is due to the more robust modulation and coding scheme used by the $48 \mathrm{Mbps}$ data rate. When activating the $802.11 \mathrm{~g}$ adaptive schemes, the critical distance was equal to 156 meters and 52 meters for the LSPE Sig function under LOS and NLOS conditions respectively. It is interesting noting that the critical distance with active adaptive schemes is higher for LOS conditions than when using the $48 \mathrm{Mbps}$ data rate, but is smaller under NLOS conditions. The different pattern could be due to the challenging NLOS propagation conditions that difficult a successful operation of the $802.11 \mathrm{~g}$ adaptive schemes. 


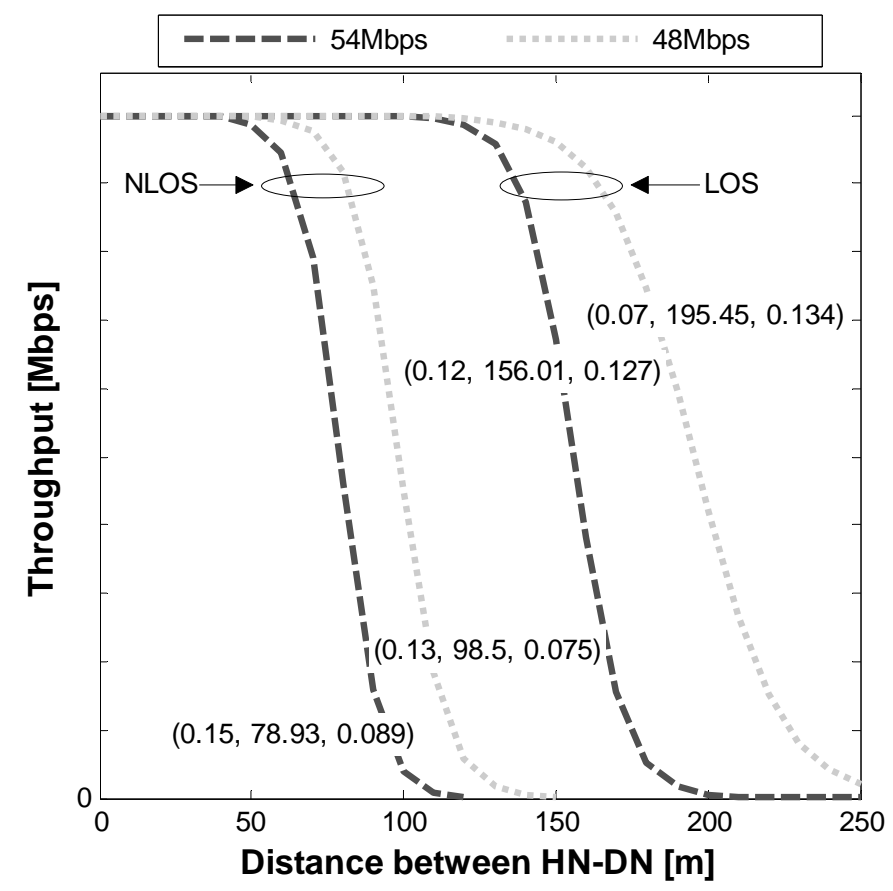

Fig. 7. 2-hop MCN throughput models at the DN with fixed IEEE 802.11g transmission modes for LOS and NLOS conditions and a transmission power of $19 \mathrm{dBm}$. The figure shows for each configuration the fitting parameters and the average residue $(p 1, p 2,\langle e\rangle)$.

\section{3-Hop MCN Communications}

This section derives models of the communications performance of 3-hop MCN in the scenario illustrated in Fig. 8. The 3-hops scenario considers the presence of an additional mobile node (Intermediate Node, IN) located between the HN and the DN. The mobile IN acts as a relay and forwards the packets coming from the HN to the DN through its IEEE 802.11g D2D link. The HN-IN and IN-DN IEEE 802.11g D2D links have been set up by modifying the routing tables of each mobile node. The HN remains in a static position during the tests, whereas the DN is a mobile node. The IN would be either static or mobile. The 3-hop MCN communication models need to take into account the possibility that the HN-IN and IN-DN links operate under LOS or NLOS conditions.

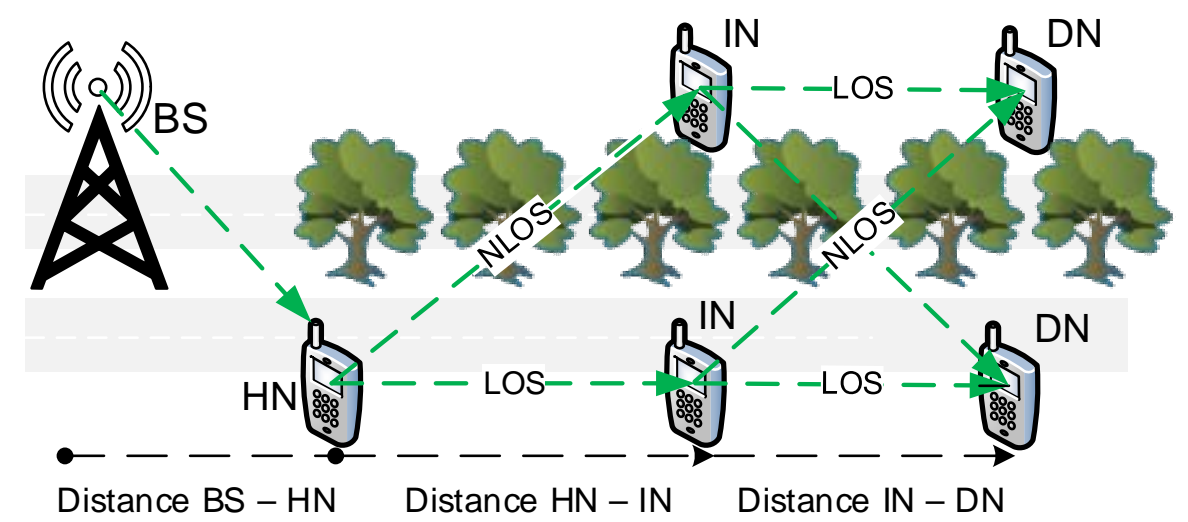

Fig. 8. Testing environment for 3-hop MCN communications. 


\subsection{Field Tests}

The 3-hop MCN performance observed at the DN does not only depend on the real-time data forwarding process from the $\mathrm{HN}$ to the $\mathrm{IN}$, but also on the decode-and-forward capability of the IN. In this context, it is important that the field tests, and consequent performance models, thoroughly analyze the impact of the mobile IN on the end-to-end MCN performance. To this aim, this section analyzes the MCN performance at the IN and the DN under diverse configurations depending on the propagation conditions (LOS and NLOS) and the distance between mobile nodes.

\subsubsection{MCN throughput at the IN}

The first tests analyzed the MCN performance at the IN under LOS and NLOS conditions with the HN. During the tests, the DN is located in the same sidewalk as the IN and they both move away from the location of the $\mathrm{HN}$ while maintaining a separation distance of approximately 30 meters. This short separation distance was selected to minimize the impact of the IN-DN link on the performance measured at the IN (the D2D connection experienced a negligible PER level and used consistently a $54 \mathrm{Mbps}$ data rate). Fig. 9 shows an example of the MCN throughput measured at the IN with the HN located 500 meters away from the BS (additional field tests were conducted for different distances between BS and HN). The measurements reported in Fig. 9 show that the MCN throughput measured at the IN initially exhibits similar performance levels to those experienced by the HN. As the IN walks away from the location of the $\mathrm{HN}$ and the HN-IN link quality degrades, the MCN throughput at the IN decreases. As expected, the throughput degradation starts at smaller distances to the HN under NLOS conditions than under LOS conditions. The distance at which the degradation starts is the IN critical distance. For the measurements reported in Fig. 9, the IN critical distance at which the IN performance decreases below the throughput performance at the $\mathrm{HN}$ is 154 and 54 meters under LOS and NLOS conditions respectively. Fig. 9 does not represent the throughput measured at the DN. However, the throughput was very similar to that measured at the IN due to the short separation distance and the LOS conditions between the IN and DN nodes. In this context, whenever the distance between the $\mathrm{HN}$ and IN is smaller than the IN critical distance, the MCN throughput measured at the DN is again upper-bounded by the HSPA cellular performance experienced at the HN. However, when such distance is exceeded, the performance is upper-bound by the HN-IN 802.11g D2D link. 


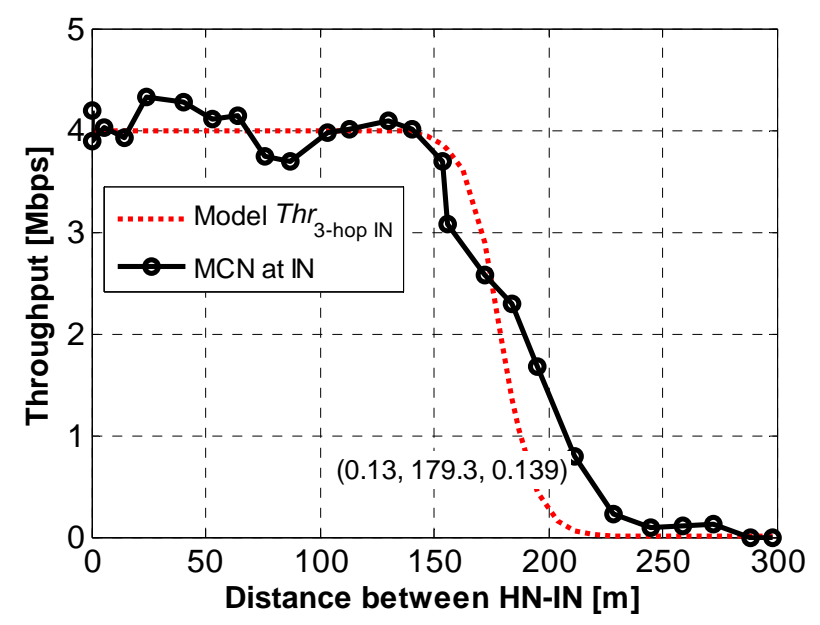

a) LOS conditions

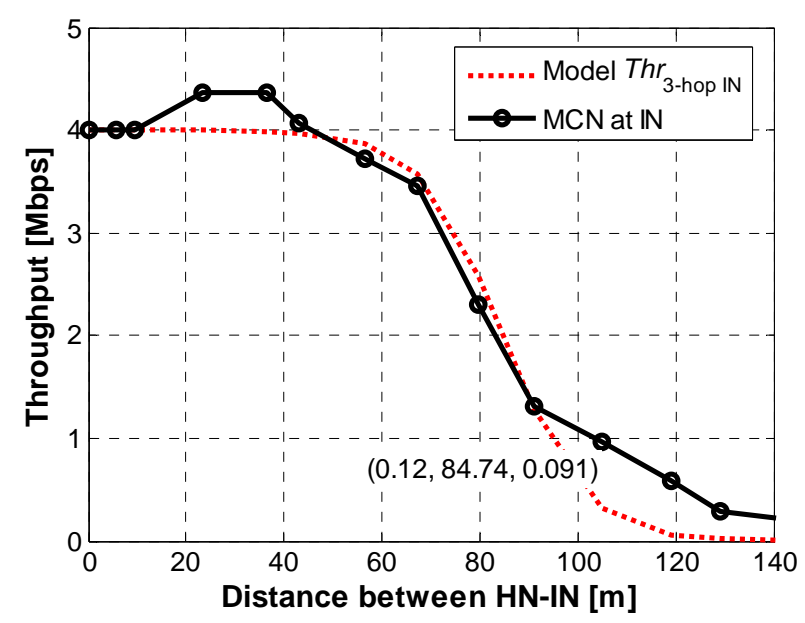

b) NLOS conditions

Fig. 9. MCN throughput measured at the IN under a 3-hop MCN configuration, and considering LOS (a) and NLOS (b) conditions between the HN and IN. The DN maintains a separation distance of $30 \mathrm{~m}$ under LOS conditions with the IN. The HN is located $500 \mathrm{~m}$ away from the BS. The figure also shows the 3-hop MCN throughput model at IN (Section 4.2). The throughput is modeled using the Sig function with the LPSE fitting parameters and residue plotted in the figures.

\subsubsection{MCN throughput at the DN}

Additional field tests were conducted to analyze the MCN performance at the DN. In this case, the $\mathrm{HN}$ and IN nodes remain static and under LOS conditions, with their separation distance being equal to 50 or 175 meters. These two values were selected to consider scenarios where the separation distance is above or below the IN critical distance. During the tests, the DN moves away from the location of the IN under LOS or NLOS conditions until the 802.11g D2D link is lost. The results depicted in Fig. 10.a show that when the HN-IN distance is shorter than the IN critical distance, the MCN throughput measured at the DN follows similar patterns as those discussed in the 2-hop scenario (Fig. 3). On the other hand, when the HN-IN distance is above the IN critical distance, the IN performance is seriously degraded (Fig. 9), and so is the 
MCN throughput measured at the DN. Fig. 10.b shows that, in this case, the MCN throughput experienced at the DN is not limited by the average HSPA throughput measured at the HN $\left(\overline{T h r_{\mathrm{HN}}}\right)$, but by the MCN throughput measured at the IN. However, it can further decrease as the distance between the IN and DN increases.
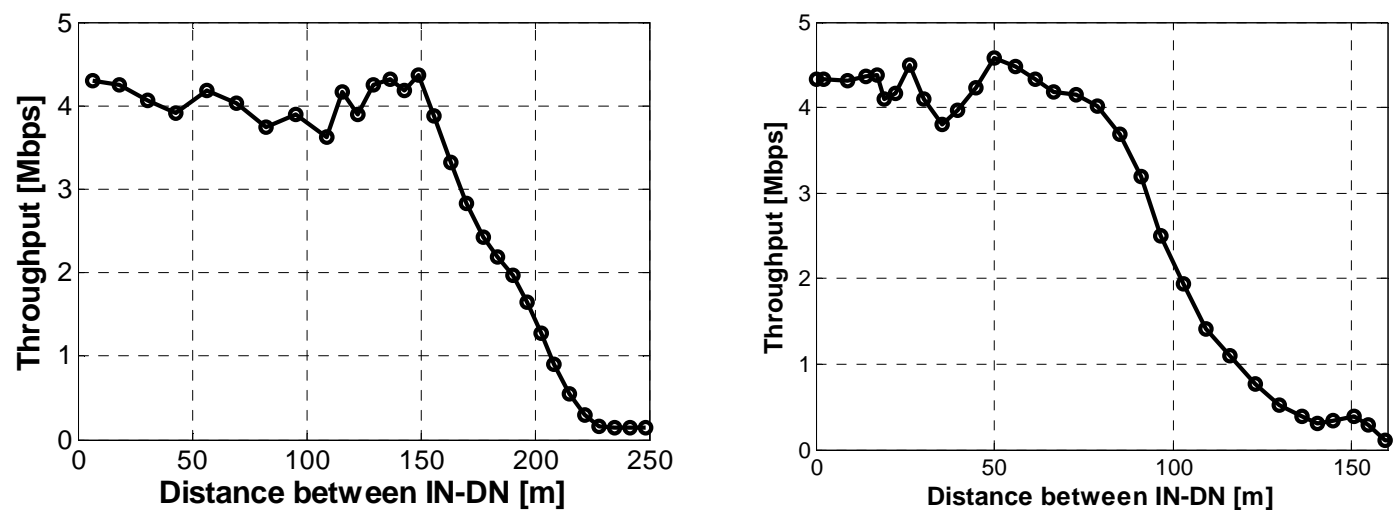

a) Distance $\mathrm{HN}-\mathrm{IN}<\mathrm{IN}$ critical distance
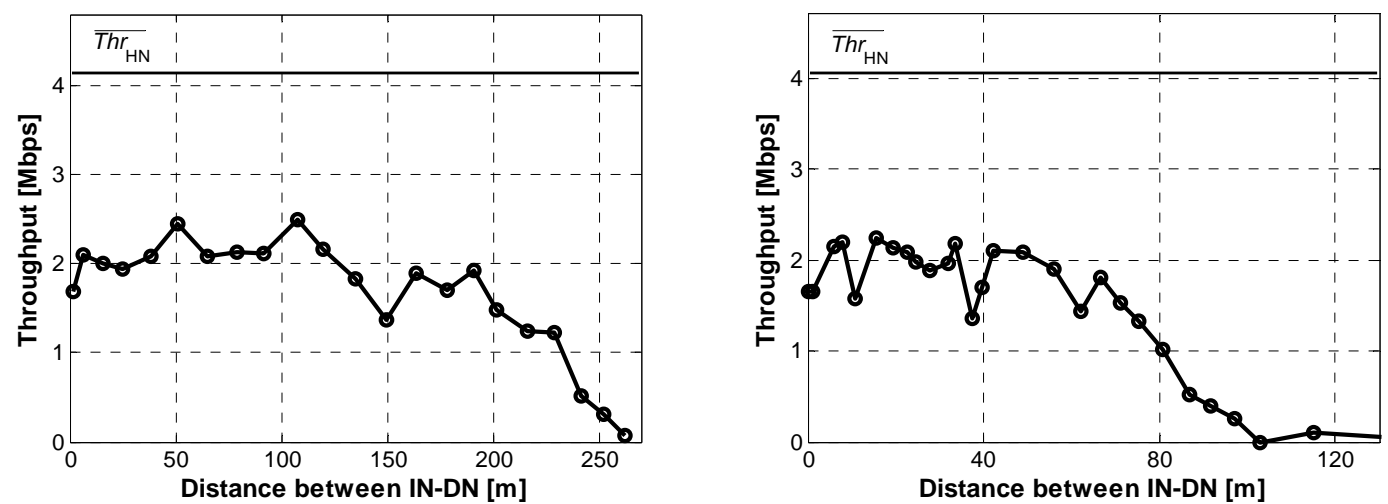

b) Distance HN-IN > IN critical distance

Fig. 10. 3-hop MCN throughput measured at the DN when the IN-DN link experiences LOS (left) and NLOS (right) conditions. The IN remains static and under LOS conditions with the HN. The HN-IN distance is 50 (a) or 175 (b) meters. The HN is located $500 m$ away from the BS.

\subsection{3-hop MCN Performance Models}

The models of the 3-hop MCN throughput performance experienced at the DN have been derived using the Sig function and the LSPE scheme described in Section 3.2. These models are represented in Fig. 11 together with the fitting parameters and average residue values $(p 1, p 2$, $\langle e\rangle)$. The 3-hop MCN throughput at the DN has been modeled as follows:

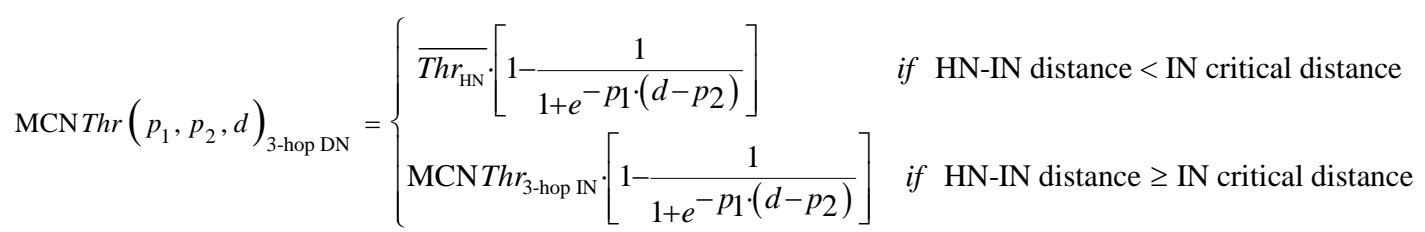

where $d$ represents the distance between IN and DN, and $\left(p_{1}, p_{2}\right)$ are the fitting parameters shown in Fig. 11. 
Previous results have shown that the MCN throughput measured at the DN is upper-bounded by the average HSPA throughput at the $\mathrm{HN}\left(\overline{T h r_{\mathrm{HN}}}\right)$ when the separation distance between the $\mathrm{HN}$ and IN nodes is smaller than the IN critical distance. When the HN-IN separation distance is higher than the IN critical distance, the MCN throughput at the DN is upper-bounded by the

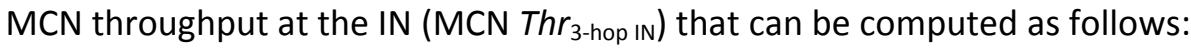

$$
\operatorname{MCN} \operatorname{Thr}\left(p_{1}, p_{2}, d\right)_{3-\mathrm{hop} \mathrm{IN}}=\overline{\operatorname{Thr}_{\mathrm{HN}}} \cdot\left[1-\frac{1}{1+e^{-p_{1} \cdot\left(d-p_{2}\right)}}\right]
$$

where $d$ represents the distance between $\mathrm{HN}$ and IN, $\left(p_{1}, p_{2}\right)$ the fitting parameters reported in Fig. 9, and $\overline{T h r_{\mathrm{HN}}}$ the average HSPA throughput at the HN that can be derived from the measurements reported in Fig. 5 and modeled in (13). For these fitting parameters, the IN critical distance at which the IN performance decreases below $\overline{T h r_{\mathrm{HN}}}$ is 154 and 54 meters under LOS and NLOS conditions respectively.

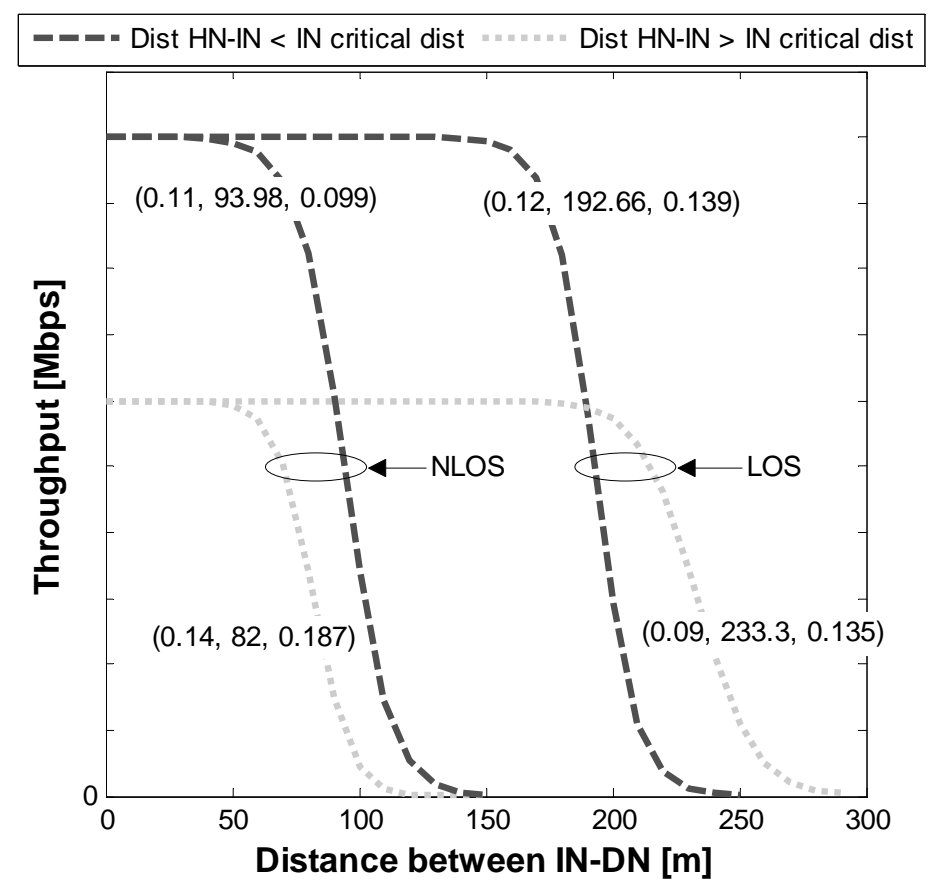

Fig. 11. 3-hop MCN throughput models at the DN when the IN-DN link experiences LOS and NLOS conditions, and for HN-IN distances smaller $(50 \mathrm{~m})$ and higher $(175 \mathrm{~m})$ than the IN critical distance. The models are derived with IN static and under LOS conditions with the HN.

The conducted study has shown that when the distance between $\mathrm{HN}$ and IN is smaller than the IN critical distance, the 3-hop MCN performance at the DN is not significantly influenced by the presence of the IN node. In fact, the fitting $\left(p_{1}, p_{2}\right)$ parameters for the MCN throughput at the DN are similar to those derived for 2-hop MCN communications (Table 2). Using the 2-hop MCN fitting parameters to model 3-hop MCN communications when the HN-IN distance is smaller than the IN critical distance will only marginally increase the average residue (approximately 7\%). On the other hand, when the distance between HN and IN is larger than the IN critical distance, the fitting parameters significantly differ, in particular under LOS conditions. An analysis of the measurements suggests that the main reason for the lower $p_{1}$ (related to the slope of the curve) and higher $p_{2}$ parameters (related to the distance at which the curve is symmetric) is the challenging $\mathrm{HN}$-IN link quality conditions that result in the IN 
using more robust transmission modes. Under NLOS conditions, the adaptive link level techniques do not perform as well as under LOS conditions, which results in the NLOS fitting $\left(p_{1}, p_{2}\right)$ parameters being similar to those derived for the 2-hop MCN communications.

\section{5. x-hop MCN Communications}

The previous sections have derived MCN performance models for likely deployment scenarios with 2 and 3 hops. A higher number of hops would increase the probability to have an unstable MCN connection as a result of a higher probability that at least one of the D2D links experiences bad link quality conditions [18]. MCN connections with a high number of hops would hence be mainly attractive if good link quality conditions can be guaranteed for the D2D links. In fact, the previous sections showed that if the distance between relay nodes was shorter than their critical distance, the HN-IN and IN-DN D2D performance was superior to the HSPA cellular performance measured at the HN. In this case, the MCN performance at the DN would be at its highest level and would be upper-bounded by the cellular performance ${ }^{8}$. It is then of interest analyzing whether this performance level and model could be extended to a larger number of hops, and the conditions under which such extension would be feasible. In this context, it is important noting that even if the D2D link quality is good, the processing at each intermediate relay decreases the multi-hop D2D performance, which could actually decrease below the cellular performance at the $\mathrm{HN}$. It is then of interest determining the maximum number of hops that would guarantee that the multi-hop ad-hoc D2D performance (and thereby the end-to-end MCN performance) is upper-bounded by the cellular performance at the HN. To this aim, we can use the channel efficiency $E$ of an IEEE 802.11 ad-hoc D2D link defined in [19] as follows:

$$
E=\frac{t_{d}}{D I F S+t_{\text {cont }}+t_{d}+S I F S+t_{a c k}}
$$

where $t_{d}, t_{\text {cont }}$ and $t_{a c k}$ represent the transmission time of the data packet, the contention period and the transmission time of the ACK frame, respectively. DIFS represents the period of time that the wireless channel has to be sensed as idle to initiate the transmission. SIFS represents the time interval between the reception of the data frame and the transmission of the ACK. DIFS and SIFS are equal to $28 \mu \mathrm{s}$ and $10 \mu \mathrm{s}$ for IEEE $802.11 \mathrm{~g}$ [20]. The value of $t_{\text {cont }}$ has been set to $67.5 \mu$ s following the indications reported in [19].

Let's suppose that the $802.11 \mathrm{~g}$ D2D links operate with the $54 \mathrm{Mbps}$ data rate ${ }^{9}$, and the size of the data packets and the ACK frame ${ }^{10}$ is 1548 bytes and 14 bytes respectively. In this case, the efficiency $E$ of an D2D link would be equal to 0.675 , which results in a maximum throughput for a single hop $802.11 \mathrm{~g}$ D2D link of $36.48 \mathrm{Mbps}$. We can then extend the efficiency

\footnotetext{
${ }^{8}$ In addition, the results showed than in this scenario the 3-hop MCN performance at the DN can be modeled using the 2-hop MCN model and its fitting parameters, with only a small residue.

${ }^{9}$ In this case, the efficiency would represent the best case scenario. However, as previously discussed, MCN connections with a high number of hops should try guaranteeing good link quality conditions for D2D connections. When this was the case, the measurements showed that the D2D links mainly operated with the $54 \mathrm{Mbps}$ data rate.

${ }^{10}$ In the conducted measurements, the ACK was transmitted at $24 \mathrm{Mbps}$.
} 
computation to various hops $E_{\text {nhops. }}$ In this case, it would be possible to identify the maximum number of hops that guarantees an end-to-end multi-hop performance higher than the cellular performance at the HN. To estimate the efficiency of a multi-hop ( $n$-hop) ad-hoc D2D link, we assume that nodes cannot transmit simultaneously over the link ${ }^{11}$. In this case, the $E_{\text {nhops }}$ efficiency needs to consider the processing time introduced by intermediate relay nodes $\left(t_{\text {pro }}\right)$. Such time has been extracted from the 3-hop MCN trials that included the intermediate IN node. $t_{\text {pro }}$ measures the time difference at the IN between the reception (from the $\mathrm{HN}$ ) and the transmission (to the DN) of 802.11 data packets. Table 5 summarizes the obtained results considering the LOS or NLOS conditions experienced at the IN-DN link. The results differentiate the measurements obtained when the HN-IN distance was higher or smaller than the IN critical distance. Several reasons explain the higher processing delay at the IN when the distance was above the IN critical distance: lower data rates, higher retransmission rates, an increase in the back-off/contention timers at the MAC layer, and the overflow of the transmission/reception buffers, among others. The channel efficiency of $n$ multi-hops ad-hoc D2D link ( $\left.E_{n h o p s}\right)$ can be computed as follows:

$$
E_{\text {nhops }}=\frac{t_{d}}{\left(\text { DIFS }+t_{\text {cont }}+t_{d}+S I F S+t_{a c k}\right)+(n-1) \cdot t_{\text {pro }}}
$$

Assuming that the distance between nodes is shorter than their critical distance, and using the average processing time values reported in Table 5, the channel efficiency is equal to $E_{\text {nhops }}=0.2255$ for a 2-hop 802.11g D2D link (3-hop MCN), $E_{\text {nhops }}=0.1353$ for a 3-hop 802.11g D2D link, and $E_{\text {nhops }}=0.0967$ for a 4-hop 802.11g D2D link. This results in a maximum throughput for a 2-hop 802.11g D2D link of 12.17Mbps, a maximum throughput for a 3-hop 802.11g D2D link of 7.31Mbps, and a maximum throughput for a 4-hop 802.11g D2D link of 5.22Mbps. The HSPA throughput measurements showed maximum values between 5 and $6.5 \mathrm{Mbps}$. In this case, a MCN connection with up to 4802.11 D2D hops could be upper-bounded by the HSPA cellular performance at the HN. Taking into account this result and the fact that a higher number of hops can result in unstable MCN connections, this section analyzes and models the performance of 4 and 5 hops MCN connections with the separation distance between the relay nodes being shorter than their critical distance.

Table 5. Average processing delay at the intermediate IN node

\begin{tabular}{c|c|c|c}
\hline \hline \multicolumn{2}{c|}{ IN-DN link under LOS } & \multicolumn{2}{c}{ IN-DN link under NLOS } \\
\hline HN-IN dist < IN critical dist & HN-IN dist > IN critical dist & HN-IN dist < IN critical dist & HN-IN dist > IN critical dist \\
\hline $0.67 \mathrm{~ms}$ & $8.6 \mathrm{~ms}$ & $0.77 \mathrm{~ms}$ & $7.2 \mathrm{~ms}$ \\
\hline \hline
\end{tabular}

\subsection{4-hop and 5-hop MCN Field Tests and Performance Models}

The performance of 4-hop and 5-hop MCN connections has been tested under similar conditions to those described for the 2-hop and 3-hop MCN scenarios. In the case of the 4-hop MCN connection, two intermediate $M N s\left(I N_{1}\right.$ and $\left.I N_{2}\right)$ are located between the $H N$ and the DN. Three INs $\left(\mathrm{IN}_{1}, \mathrm{IN}_{2}\right.$ and $\left.I \mathrm{IN}_{3}\right)$ are needed for the 5-hop MCN connection. The HN and INs remain

11 This assumption results in a lower-bound of the multi-hop channel efficiency since a single data packet can be transmitted at each point in time over the multi-hop link. 
static during the field tests, and they maintain a separation distance of 50 meters ${ }^{12}$ under LOS conditions (this distance is below the critical distance of each D2D link). The DN moves away from the location of its closer IN (IN $\mathrm{IN}_{2}$ for 4-hop MCN and $\mathrm{IN}_{3}$ for 5-hop MCN) under LOS or NLOS conditions. The obtained results showed similar trends to that analyzed previously for 2hop and 3-hop MCN communications. The 4-hop and 5-hop MCN performance models have hence been derived using the Sig function and the LSPE fitting process, and can be expressed as:

$$
\operatorname{MCN} \operatorname{Thr}\left(p_{1}, p_{2}, d\right)_{4-\text { hop or 5-hop DN }}=\overline{T h r_{\mathrm{HN}}} \cdot\left[1-\frac{1}{1+e^{-p_{1} \cdot\left(d-p_{2}\right)}}\right]
$$

where $d$ represents the distance between $\mathrm{IN}_{2}$ and $\mathrm{DN}$ for the 4-hop scenario, and the distance between $\mathrm{IN}_{3}$ and $\mathrm{DN}$ for the 5-hop scenario. The models together with the fitting parameters and average residues $(p 1, p 2,\langle e\rangle)$ for the two scenarios are plotted in Figure 12 and 13 respectively. The average residues for 4 -hop MCN communications increase about $17 \%$ and $25 \%$ under LOS and NLOS conditions compared to the average residues obtained for 2-hop MCN communications. The increment for the 5-hop MCN scenario compared to the 2-hop MCN one is equal to $25 \%$ and $30 \%$ under LOS and NLOS. The obtained results show that MCN connections with a high number of hops can still be attractive if good link quality conditions are guaranteed for the D2D links. In fact, the average residues for 4-hop and 5-hop MCN communications is smaller under the considered conditions that with the 3-hop MCN scenario when the distance between IN and DN was higher than the IN critical distance.

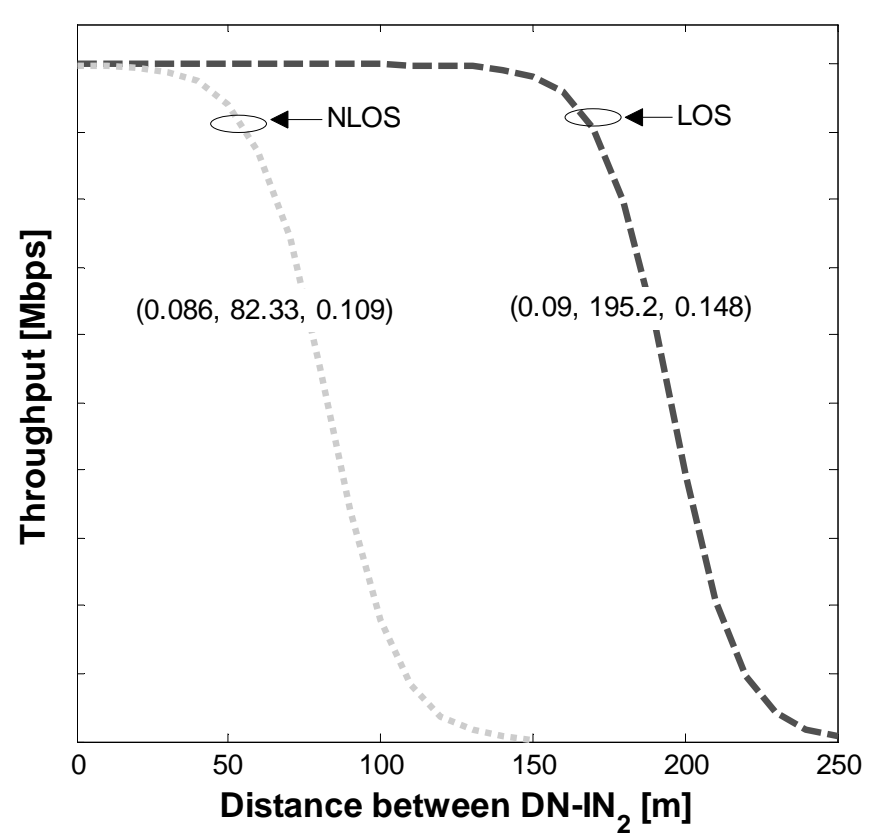

Fig. 12. MCN throughput model at the DN under a 4-hop MCN configuration, and considering LOS and NLOS condition between $\mathrm{IN}_{2}$ and DN.

\footnotetext{
${ }^{12}$ The scenario where the distance between relay nodes is higher than their critical distance has not been reproduced for 4-hop and 5-hop MCN communications. This was decided following the discussion about the need to guarantee D2D links with good link quality conditions in order to ensure stable multihop MCN connections.
} 


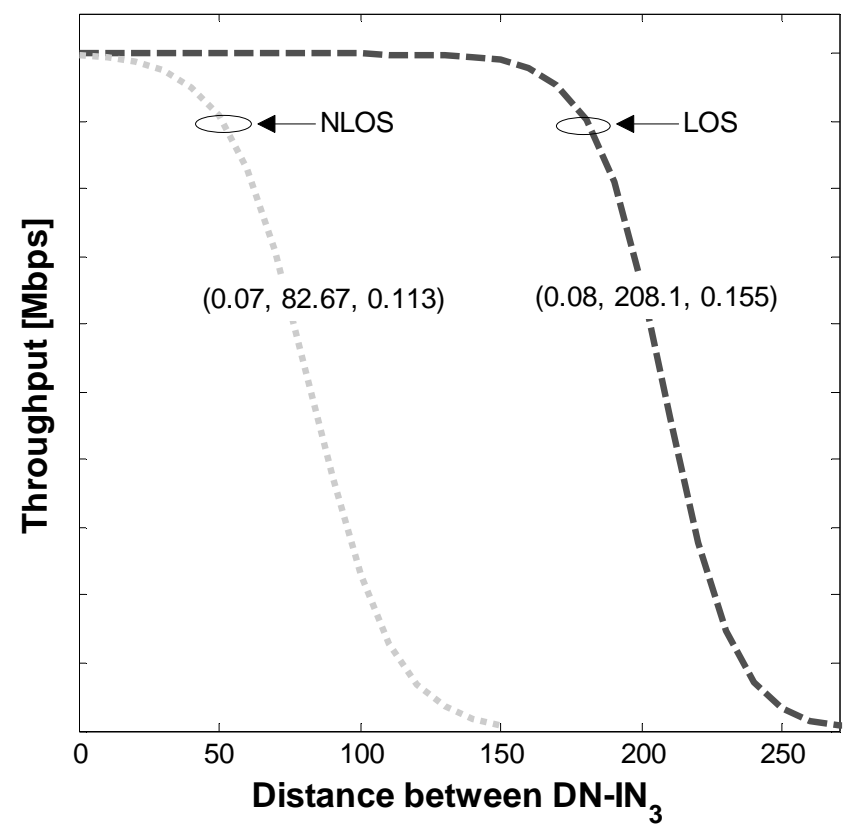

Fig. 13. MCN throughput model at the DN under a 5-hop MCN configuration, and considering LOS and NLOS conditions between $\mathrm{IN}_{3}$ and $\mathrm{DN}$.

\section{Conclusions}

This study has presented novel and unique models for the communications performance of multi-hop cellular networks using mobile relays and D2D communications. The proposed models can be significantly valuable to support the design, test and optimization of novel communications and networking protocols tailored for MCNs. The models have been obtained following a large field testing campaign using an active cellular network in the city of Elche in Spain. The reported models represent the MCN throughput experienced at a mobile destination node as a function of the distance, propagation/visibility conditions, number of hops and communication settings. The performance metric was selected with the objective to reduce the impact of cellular resource management policies that may vary from operator to operator. The MCN throughput performance has been modeled with a sigmoid function that is a function of the cellular throughput experienced at the hybrid or gateway mobile node. The models' fitting parameters have been shown to depend on key factors such as the propagation conditions and number of hops. The models have been derived considering HSPA cellular transmissions and IEEE 802.11g D2D communications. Extending the models to other cellular technologies could be feasible as long as the MCN performance is upper-bounded by the cellular link between the BS and the hybrid mobile node.

\section{Acknowledgements}

The authors would like to thank Prof. Joaquin Sanchez-Soriano from UMH's Operation Research Center for his guidance during the modeling process. This work is supported in part by the Spanish Ministry of Economy and Competitiveness and FEDER funds under the projects TEC2008-06728 and TEC2011-26109, and the Local Government of Valencia with reference ACIF/2010/161. The authors also acknowledge the support of Orange Spain. 


\section{References}

[1] A. Osseiran, F. Boccardi, V. Braun, K. Kusume, P. Marsch, M. Maternia, O. Queseth, M. Schellmann, H. Schotten, H. Taoka, H. Tullberg, M.A. Uusitalo, B. Timus, and M. Fallgren, "Scenarios for $5 \mathrm{G}$ mobile and wireless communications: the vision of the METIS project", IEEE Communications Magazine, vol. 52, no. 5, pp. 26-35, May 2014 . DOI: 10.1109/MCOM.2014.6815890.

[2] F. Boccardi, R.W. Heath, A. Lozano, T.L. Marzetta and P. Popovski, "Five Disruptive Technology Directions for 5G", IEEE Communications Magazine, vol. 52, no. 2, pp. 74-80, Feb. 2014. DOI: 10.1109/MCOM.2014.6736746.

[3] J. Gozalvez and B. Coll-Perales, "Experimental Evaluation of Multi-Hop Cellular Networks using Mobile Relays", IEEE Communications Magazine, vol. 51, no. 7, pp. 122-129, July 2013. DOI: 10.1109/MCOM.2013.6553688.

[4] 3GPP TR 22.803 V12.1.0. 2013. Technical Specification Group Services and System Aspects; Feasibility study for Proximity Services (ProSe). Mar. 2013.

[5] A. Catovic and S. Tekinay, "Power Efficiency of User Cooperation in Multihop Wireless Networks", IEEE Communications Letters, vol. 9, no. 12, pp. 1034-1036, Dec. 2005. DOI: 10.1109/LCOMM.2005.1576579.

[6] N. Akkari, G. Aldabbagh, M. Nahas and John Cioffi, "Dynamic Clustering Protocol for coordinated tethering over cellular networks", Journal of Network and Computer Applications, vol. 42, no. 6, pp. 92-101, Jun. 2014. DOI: 10.1016/j.jnca.2014.03.011.

[7] B. Coll-Perales and J. Gozalvez, "On the Capability of Multi-hop Cellular Networks with Mobile Relays to Improve Handover Performance", Proc. 8th IEEE International Symposium on Wireless Communication Systems (ISWCS), pp. 207-211, Nov. 2011, doi: 10.1109/ISWCS.2011.6125339.

[8] M.C. Lucas-Estañ, J. Gozalvez and B. Coll-Perales, "Mode Selection for Mobile Opportunistic Multi-Hop Cellular Networks", Proc. 79th IEEE Vehicular Technology Conference (VTC2014Spring), May 2014.

[9] R. Ananthapadmanabha, B.S. Manoj and C.S.R. Murthy, "Multi-hop Cellular Networks: the Architecture and Routing Protocols", Proc. 12th IEEE International Symposium on Personal, Indoor and Mobile Radio Communications (PIMRC), pp. 78-82, Sep/Oct 2001. DOI: 10.1109/PIMRC.2001.965324.

[10] B. Coll-Perales and J. Gozalvez, "Energy Efficient Routing Protocols for Multi-hop Cellular Networks", Proc. 20th IEEE International Symposium on Personal, Indoor and Mobile Radio Communications (PIMRC), pp.1457-1461, Sept. 2009. DOI: 10.1109/PIMRC.2009.5450170.

[11] Q. Ayub, S. Rashid, M.S. Mohd-Zahid and A.H. Abdullah, "Contact quality based forwarding strategy for delay tolerant network", Journal of Network and Computer Applications, vol. 39, no. 3, pp. 302-309, Mar. 2014. DOI: 10.1016/j.jnca.2013.07.011.

[12] B. Coll-Perales, J. Gozalvez and V. Friderikos, "Opportunistic Networking for Improving the Energy Efficiency of Multi-Hop Cellular Networks", Proc. 11th Annual IEEE Consumer Communications \& Networking Conference (CCNC 2014), Jan. 2014. DOI: 10.1109/CCNC.2014.6866628.

[13] P. Mahasukhon, M. Hempel, C. Song and H. Sharif, "Comparison of Throughput Performance for the IEEE 802.11a and 802.11g Networks", Proc. 21st IEEE International Conference on Advanced Information Networking and Applications (AINA), pp. 792-799, May 2007. DOI: 10.1109/AINA.2007.46.

[14] B. Coll-Perales, J. Gozalvez and J. Sánchez-Soriano, "Empirical Performance Models for P2P and Two Hops Multi-hop Cellular Networks with Mobile Relays", Proc. 8th ACM Workshop on Performance Monitoring, Measurements and Evaluation of Heterogeneous Wireless and Wired Networks (PM2HW2N-2013), pp. 21-28, Nov. 2013. DOI: 10.1145/2512840.2512844.

[15] D. Krishnaswamy, "Game Theoretic Formulations for Network-Assisted Resource Management in Wireless Networks", Proc. 56th IEEE Vehicular Technology Conference (VTC 2002-Fall), pp. 1312-1316, Sept. 2002. DOI: 10.1109/VETECF.2002.1040428.

[16] C. Moler, Numerical Computing with Matlab. Ed. SIAM, pp. 155-182, 2004.

[17] TradersPlace, "Moving Averages in Technical Analysis", WhitePaper available online on 2014.

[18] C. Aydogdu and E. Karasan, "An Analysis of IEEE 802.11 DCF and Its Application to EnergyEfficient Relaying in Multihop Wireless Networks", IEEE Trans. on Mobile Computing, vol. 10, 
no. 10, pp. 1361-1373, Oct. 2011, doi: 10.1109/TMC.2010.239.

[19] A. Duda, "Understanding the Performance of 802.11 Networks", Proc. 19th IEEE International Symposium on Personal, Indoor and Mobile Radio Communications (PIMRC), pp. 1-6, Sept. 2008, doi: 10.1109/PIMRC.2008.4699942.

[20] IEEE 802.11g, "Amendment 4: Further Higher Data Rate Extension in the $2.4 \mathrm{GHz}$ Band,"IEEE Standard, 2003.

B. Coll-Perales received a Telecommunications Engineering degree from the Miguel Hernandez University (UMH) of Elche (Spain) in 2008. He received Best Student awards in Telecommunications Engineering both by $\mathrm{UMH}$ and the professional organization of Telecommunications Engineers. He then joined the UWICORE re-search laboratory to work on the development of networking and communication protocols for multi-hop cellular systems using mobile relays under the $\mathrm{m}$-HOP and ICARUS projects. He is currently pursuing his PhD studies with a fellowship from the Valencia Regional government. His research focuses on multi-hop cellular networks, including networking, connectivity and resource management aspects.

Javier Gozalvez received an electronics engineering degree from the Engineering School ENSEIRB (Bordeaux, France), and a PhD in mobile communications from the University of Strathclyde, Glasgow, U.K. Since October 2002, he is with the Miguel Hernández University of Elche, Spain, where he is currently an Associate Professor and Director of the UWICORE laboratory. At UWICORE, he leads research activities in the areas of multi-hop cellular networks, vehicular networks, resource management and heterogeneous networks, and wireless industrial communications. He has published over 110 papers in international conferences and journals. He is an elected member to the Board of Governors (2011-2017) and Executive Vice President of the IEEE Vehicular Technology Society. He is an IEEE Distinguished Lecturer for the IEEE Vehicular Technology Society. He currently serves as Mobile Radio Senior Editor of IEEE Vehicular Technology Magazine, and previously served as AE of IEEE Communication Letters. He is the General Co-Chair for the IEEE VTC-Spring 2015 conference in Glasgow (UK), and was General Co-Chair of the ACM VANET 2013, ACM VANET 2012 and 3rd ISWCS 2006. He also was TPC Co-Chair for 2011 IEEE VTC-Fall and 2009 IEEE VTC-Spring. He is also the founder and General Co-Chair of the IEEE International Symposium on Wireless Vehicular communications (WiVeC) in its 2007, 2008, and 2010 editions.

Miguel Sepulcre received a telecommunications engineering degree in 2004 and a Ph.D. in communications technologies in 2010, both from the Miguel Hernández University of Elche (UMH), Spain. In 2004, he spent six months at the European Space Agency (ESA) in Noordwijk (The Netherlands) working on the communications physical layer of earth exploration satellites. In 2006, he obtained a Ph.D. fellowship from the Valencian regional government and joined the UWICORE research laboratory to work on vehicular communications. He was awarded by the COIT (Spanish official association of Telecommunication Engineers) with the ONO prize to the best Ph.D. thesis. He is currently a research fellow at the UWICORE Laboratory of $\mathrm{UMH}$, working on vehicular communications, radio resource management, modelling and simulation of wireless communications systems. 